\title{
Probing ice clouds by broadband mid-infrared extinction spectroscopy: case studies from ice nucleation experiments in the AIDA aerosol and cloud chamber
}

\author{
R. Wagner, S. Benz, O. Möhler, H. Saathoff, and U. Schurath \\ Forschungszentrum Karlsruhe, Institute of Meteorology and Climate Research, Karlsruhe, Germany
}

Received: 20 April 2006 - Published in Atmos. Chem. Phys. Discuss.: 3 July 2006

Revised: 7 September 2006 - Accepted: 19 September 2006 - Published: 24 October 2006

\begin{abstract}
Series of infrared extinction spectra of ice crystals were recorded in the $6000-800 \mathrm{~cm}^{-1}$ wavenumber regime during expansion cooling experiments in the large aerosol and cloud chamber AIDA of Forschungszentrum Karlsruhe. Either supercooled sulphuric acid solution droplets or dry mineral dust particles were added as seed aerosols to initiate ice formation after having established ice supersaturated conditions inside the chamber. The various ice nucleation runs were conducted at temperatures between 237 and $195 \mathrm{~K}$, leading to median sizes of the nucleated ice particles of 1-15 $\mu \mathrm{m}$. The measured infrared spectra were fitted with reference spectra from $\mathbf{T}$-matrix calculations to retrieve the number concentration as well as the number size distribution of the generated ice clouds. The precise evaluation of the time-dependent ice particle number concentrations, i.e., the rates of new ice particle formation, is of particular importance to quantitatively analyse the ice nucleation experiments in terms of nucleation rates and ice activation spectra. The ice particles were modelled as finite circular cylinders with aspect ratios ranging from 0.5 to 3.0. Benefiting from the comprehensive diagnostic tools for the characterisation of ice clouds which are available at the AIDA facility, the infrared retrieval results with regard to the ice particle number concentration could be compared to independent measurements with various optical particle counters. This provided a unique chance to quantitatively assess potential errors or solution ambiguities in the retrieval procedure which mainly originate from the difficulty to find an appropriate shape representation for the aspherical particle habits of the ice crystals. Based on these inter-comparisons, we demonstrate that there is no standard retrieval approach which can be routinely applied to all different experimental scenarios. In particular, the concept to account for the asphericity of the ice crystals, the a priori constraints which might be imposed
\end{abstract}

Correspondence to: R. Wagner

(robert.wagner@imk.fzk.de) on the unknown number size distribution of the ice crystals (like employing an analytical distribution function), and the wavenumber range which is included in the fitting algorithm should be carefully adjusted to each single retrieval problem.

\section{Introduction}

Due to the important impact of ice crystal clouds (cirrus) on the earth-atmosphere radiation balance, numerous field and laboratory studies have been conducted to explore and to quantify the two distinct freezing mechanisms that lead to the formation of the ice phase: (i) homogeneous freezing of aqueous solution droplets, and (ii) heterogeneous ice nucleation by solid materials, so-called ice nuclei (Lynch et al., 2002). For this purpose, field missions, like the CRYSTAL-FACE measurement campaign on tropical cirrus cloud physical properties and formation processes, are featuring extensive instrumentation for the characterisation of both the aerosol and the ice particles (http:// cloud1.arc.nasa.gov/crystalface/instruments.html). Amongst simultaneous laboratory studies on the freezing properties of aerosols prevalent in the troposphere, the ice nucleation experiments at the AIDA aerosol and cloud chamber facility of Forschungszentrum Karlsruhe also benefit from excellent diagnostic techniques for the characterisation of ice clouds (Wagner et al., 2006). Thereby, cloud microphysical processes can be analysed in a quantitative manner, as e.g. recently demonstrated by measuring T-dependent homogeneous ice nucleation rates in supercooled cloud droplets (Benz et al., 2005) or by quantifying the efficiency of heterogeneous ice nucleation on mineral dust particles (Möhler et al., 2006).

A basic requirement for the quantitative analysis of the AIDA investigations is the accurate determination of the number concentration of the ice crystals which are formed in the course of ice nucleation experiments using the AIDA

Published by Copernicus GmbH on behalf of the European Geosciences Union. 
aerosol vessel as a cloud expansion chamber. In recent measurement campaigns, up to three independent devices for counting ice particles were attached to the chamber (Field et al., 2006; Möhler et al., 2006): two optical particle counters (OPCs: PCS2000 and WELAS, Palas GmbH, Germany) and the laser scattering device SID (small ice detector, developed by the University of Hertfordshire, UK). Correlation plots of the ice particle concentrations measured by the different instruments generally indicate good agreement within an uncertainty range of about 20\%; see Figs. 3 and 4 in Möhler et al. (2006). As further part of the AIDA instrumentation, broadband infrared extinction measurements are another powerful tool to study the formation and growth of ice crystals. Based on reliable infrared complex refractive indices for ice (Clapp et al., 1995; Zasetsky et al., 2005), details of the size distribution of the ice crystals as well as the ice water content of a cloud may be retrieved from the measured infrared spectra. As infrared measurements are also an important remote sensing technique for the analysis of ice clouds (e.g. Eremenko et al., 2005; Kahn et al., 2003, 2004; Rädel et al., 2003), the literature already offers a wealth of information on the scattering and absorption properties of cirrus clouds at infrared wavelengths. For example, Baran (2004) has recently summarised concepts for representing the various shapes of ice crystals that exist in cirrus clouds as well as computational methods for calculating their single-scattering properties. As clearly shown by Liu et al. (1999), the infrared retrieval results are notably influenced by different assumptions on the shape of the ice crystals. This was demonstrated by calculating a synthetic infrared extinction spectrum for a given size distribution of finite circular cylinders of aspect ratio $\phi=1$ using the $\mathbf{T}$-matrix method and inverting the spectrum applying Mie theory. The incorrect choice of particle shape (spheres instead of cylinders) was transformed into serious errors in the retrieved size distribution, even leading to spurious modes of smaller and larger particles; see Fig. 7 in Liu et al. (1999). In contrast, the retrieved infrared optical depths closely matched the synthetic spectrum. This solution ambiguity may therefore constrain the applicability of infrared measurements for deriving reliable information about the number concentration and size distribution of ice clouds. In this context, the ice nucleation experiments at the AIDA cloud chamber, performed under realistic atmospheric conditions, may be a valuable complement, since the retrieval results from the infrared extinction spectra can be directly compared to those measured by independent techniques, i.e., to the data from the OPCs and the SID instrument.

In this work, we will present selected case studies of AIDA expansion experiments in the 237-195 K temperature regime. Ice clouds were generated both by homogeneous freezing of supercooled $\mathrm{H}_{2} \mathrm{SO}_{4} / \mathrm{H}_{2} \mathrm{O}$ solution droplets and by heterogeneous ice nucleation on mineral dust particles. Depending on the number concentration of the seed aerosol particles as well as the prevailing water vapour pressure, the ice crystals grew to median sizes of 1 to 15 microns in the course of the different expansion runs. FTIR extinction spectra of the ice particles were recorded in situ from 6000-800 wavenumbers. For retrieving the size distribution from the measured infrared spectra, the ice crystals were modelled as finite circular cylinders in random orientation with aspect ratios ranging from 0.5 to 3.0 , using the $\mathbf{T}$-matrix code for calculating the corresponding extinction cross sections (Mishchenko and Travis, 1998). Both pure cylindrical shapes and shape distributions of cylinders with different aspect ratios were used in the retrievals. By comparing the FTIR retrieval results with the simultaneously recorded data from the OPCs, we want to explore whether and to which extent infrared extinction measurements are appropriate for a reliable determination of the number concentration and size distribution of ice clouds in the given range of particle sizes.

In Sect. 2 of this paper, we will briefly review the instrumentation of the AIDA chamber and describe the experimental procedure of a typical ice nucleation experiment. A separate subsection will describe the set-up for the in situ FTIR extinction measurements as well as the retrieval algorithm to derive the size distribution of the ice crystals from the measured infrared extinction spectra. Before presenting the results of the AIDA experiments, we will show selected results of our T-matrix calculations for finite ice cylinders in Sect. 3. Plots of the extinction efficiency at absorbing and non-absorbing infrared wavelengths versus particle size for different ice crystal shapes will reveal the magnitude of the shape-related differences in ice crystal absorption and scattering. In addition, they will already point to potential solution ambiguities in the retrieval procedure as different sizeshape distributions of ice cylinders may lead to almost identical infrared extinction spectra. These a priori considerations are then illustrated by means of selected AIDA ice nucleation runs in Sect. 4. A summary of the results as well as an outlook to ongoing AIDA investigations on this subject are given in Sect. 5.

\section{Experimental}

\subsection{AIDA expansion cooling experiments}

The technical equipment of the AIDA chamber as well as its operation as an expansion cloud chamber for ice nucleation studies has been described in detail in our recent publications (Möhler et al., 2005, 2003; Wagner et al., 2006) and will only briefly be summarised here. Being insulated in a large thermostated housing, the cylindrical AIDA aerosol vessel (4 m diameter, $84.3 \mathrm{~m}^{3}$ volume) can be cooled to any temperature between ambient and $183 \mathrm{~K}$ (Fig. 1), thereby featuring spatial and temporal temperature fluctuations of less than $\pm 0.3 \mathrm{~K}$ throughout the whole chamber interior. In addition to the excellent temperature control, the AIDA chamber can also be evacuated with two vacuum pumps to less than $0.01 \mathrm{hPa}$. 


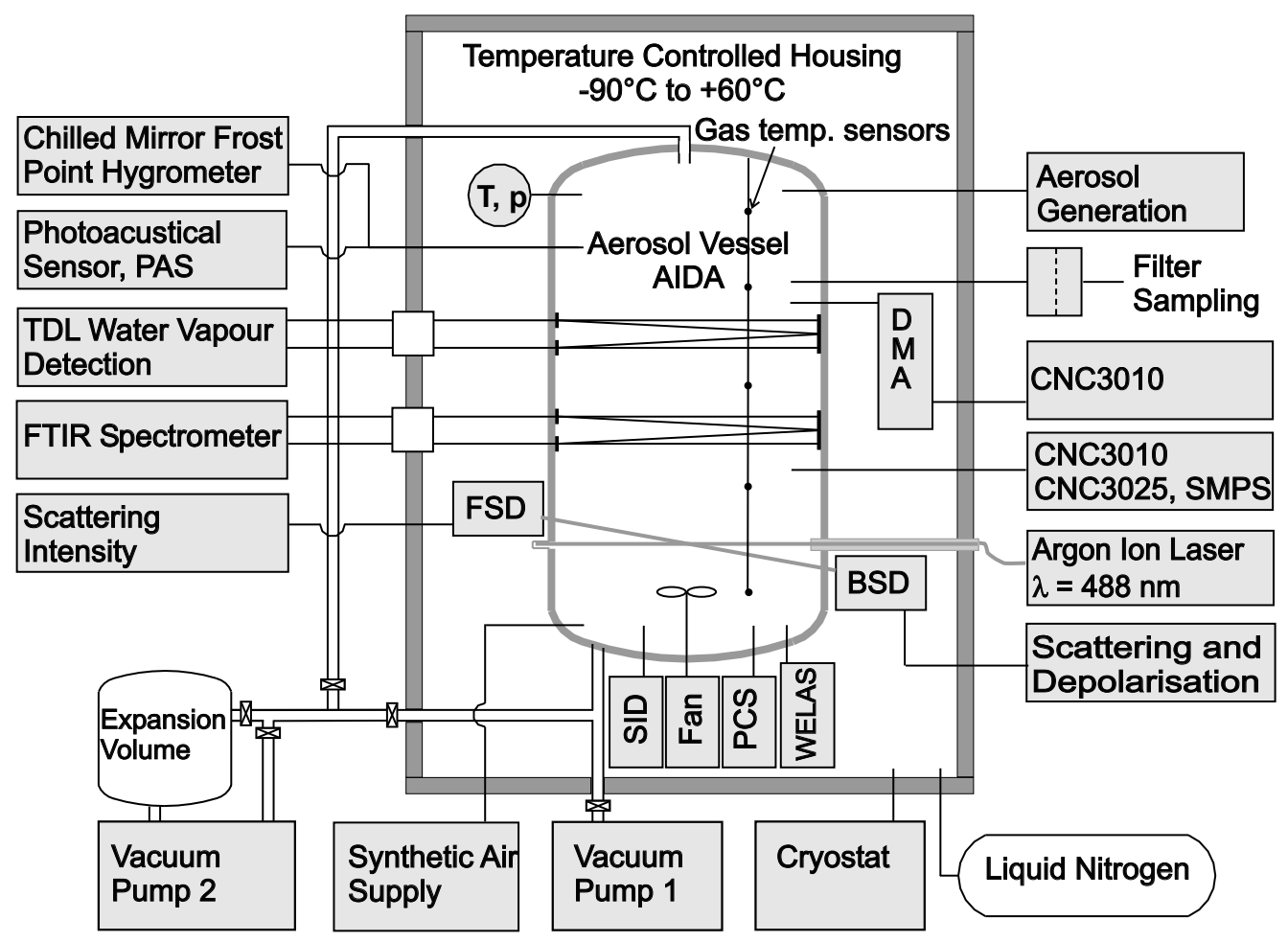

Fig. 1. Schematic representation of the AIDA aerosol and cloud chamber facility of Forschungszentrum Karlsruhe, showing the instrumentation for ice nucleation studies. A mixing fan is operating at constant speed throughout an expansion experiment. Specifications of the devices which are relevant for the present study are described in the text. The detailed set-up for the FTIR extinction measurements is separately shown in Fig. 3.

This additional pressure control provides three important opportunities for chamber experiments: (i) a highly efficient cleaning of the aerosol vessel by evacuating the chamber and repeating several flushing cycles with particle-free synthetic air, (ii) performing chamber studies at different pressure levels, (iii) establishing ice supersaturated conditions inside the chamber by controlled pumping to study ice nucleation.

A typical AIDA ice nucleation experiment is started after (i) cleaning of the aerosol vessel, (ii) coating the walls of the AIDA chamber with a thin ice layer, and (iii) addition and characterisation of the desired seed aerosol particles. The coating is made by filling the chamber volume with humidified air at a higher temperature before cooling to a lower temperature which was chosen as the starting temperature for the expansion cooling run. Due to the ice coating, an ice saturation ratio $S_{\text {ice }}(T)=p_{w}(T) / p_{w}$,ice $(T)$ close to one is established inside the chamber before the expansion is started, as calculated from the actual water vapour pressure, $p_{w}(T)$, and the saturation water vapour pressure over ice, $p_{w \text {,ice }}(T)$. To achieve supersaturations with respect to the ice phase, the pressure inside the chamber is reduced by controlled pumping, typically from $1000-800 \mathrm{hPa}$ within 4-10 min, corresponding to cooling rates of about $0.1-4 \mathrm{~K}$ $\min ^{-1}$. Thereby, ice saturation ratios greater than 1.6 can be established in the course of the expansion, as $p_{w \text {,ice }}(T)$ exponentially decreases with decreasing gas temperature, whereas $p_{w}(T)$ decreases linearly with decreasing pressure, or less than linearly in experiments with ice-coated chamber walls. Ice nucleation occurs upon exceeding a critical threshold relative humidity with respect to ice, its value strongly varying with the nature of the seed aerosol and the temperature. Until 2004, the AIDA ice nucleation studies have focussed on the determination of this critical ice saturation ratio, relying on accurate measurements of $p_{w}(T)$ using tuneable diode laser absorption spectroscopy and the precise determination of the onset of ice nucleation by measuring the increase in depolarization of back-scattered laser light; see e.g. Figs. 6 in Möhler et al. (2003) and Wagner et al. (2006). As already indicated in the introduction, data analysis of current AIDA ice nucleation studies is progressing towards a quantitative evaluation of the freezing processes, i.e., towards the determination of nucleation rates and ice activation spectra, meaning the fraction of ice activated aerosol particles as function of the ice saturation ratio; see e.g. Figs. 7 and 8 in Möhler et al. (2006). The precision of these analyses depends on the performance of the various instruments for counting and sizing ice crystals which are attached to the AIDA chamber. Their operation will be described in Sect. 2.2. In our present study, the 
cross-check with these devices is used to assess the accuracy of the retrieval results from the FTIR extinction spectra.

By the end of 2005, about 750 different expansion cooling runs have been conducted in the AIDA chamber, most of them with concomitant FTIR extinction measurements. Our intention is not to give an exhaustive overview about all these experiments, which cover a broad range of temperatures and a large variety of different seed aerosol particles. Instead, we will focus on a few, exemplary expansion runs that will underline the basic problems associated with the FTIR retrieval procedure. The expansion experiments which will be shown in this work were conducted at temperatures between 237 and $195 \mathrm{~K}$. Ice clouds were generated via homogeneous freezing of supercooled $\mathrm{H}_{2} \mathrm{SO}_{4} / \mathrm{H}_{2} \mathrm{O}$ solution droplets or via deposition ice nucleation on mineral dust particles. Desert dust samples from Asia and the Sahara as well as the so-called Arizona test dust were employed; see Möhler et al. (2006) for details. In one case, two nucleation events occurred in series, the first being due to heterogeneous ice nucleation on mineral dust particles, followed by homogeneous freezing of sulphuric acid solution droplets which were present in an external mixture. Further details of the various expansion runs are given in direct context with the discussion of the FTIR retrieval results in Sect. 4.

In natural cirrus clouds, there is a considerable variability in the size of the ice crystals, ranging from $<10 \mu \mathrm{m}$ to $>1 \mathrm{~mm}$ (Lynch et al., 2002). In the AIDA chamber studies, the size of the ice crystals is mainly determined by the number concentration of ice activated seed aerosol particles and the prevailing water vapour pressure, which, due to the ice-coated chamber walls, exponentially increases with increasing temperature. So, the higher the AIDA gas temperature and the lower the number concentration of ice activated seed aerosol particles which are competing for the available amount of water vapour, the larger are the sizes of the nucleated ice crystals. In the expansion experiments selected for this study, the ice crystals grew to sizes of about 1 to 15 microns, being representative of young contrails or highaltitude tropical cirrus clouds (Eremenko et al., 2005). In this size range, there is usually no independent information about the actual shape of the ice crystals, since commercial instruments for imaging ice crystals like the cloud particle imager CPI (Stratton Park Engineering Company, SPEC Inc.) are only able to image particles with sizes larger than about $10 \mu \mathrm{m}$ (Lawson et al., 2001). Additionally, at small sizes from about $10-15 \mu \mathrm{m}$, the resolving power of the instrument might be sufficient to categorise a particle as aspherical (i.e., it can be distinguished from a spherical cloud droplet) but not to fully resolve its actual habit. Such crystals are then usually classified as "small irregulars". Thus, the FTIR retrieval procedure (as described in Sect. 2.3) must manage without an a priori knowledge of the ice particle habit.

\subsection{Ice cloud measurements}

The number concentrations of the ice crystals which are formed during the AIDA expansion runs are measured with two OPCs (PCS2000 and WELAS, Palas GmbH, Germany) and the laser scattering device SID. The PCS2000 and WELAS instruments belong to the basis instrumentation of the AIDA chamber, the SID device was contributed by external partners from the Met Office, UK, and was available only for specific AIDA measurement campaigns. From all devices, the WELAS measurements feature the most complete data set and are primarily used for the inter-comparison with the FTIR retrievals. All particle counting instruments are mounted at the bottom of the AIDA vessel within the thermostated housing (see Fig. 1), thus are operating at chamber conditions. Strictly vertical sampling lines were used to minimise sampling losses, using flow rates of typically $51 / \mathrm{min}$. The operation of the SID device is described in detail by Field et al. (2006). Briefly, six azimuthally arranged detectors are used to detect forward scattered light at an angle of $30^{\circ}$. This detector arrangement allows for discrimination between spherical and aspherical particles. Appropriate sizing, however, is only possible for spherical particles in the $1-35 \mu \mathrm{m}$ size range. Both the PCS2000 and WELAS instruments are OPCs which measure the pulses of white light scattered by single particles into an angle of $90 \pm 12^{\circ}$ for counting and sizing; see Benz et al. (2005) for details. The smallest spherical particles that can be detected by these OPCs have diameters in the range from about $0.5-0.8 \mu \mathrm{m}$ for refractive indices between 1.5 and 1.3. Again, sizing is incorrect for aspherical particle shapes (i.e., ice crystals) as the response of the detector depends on the accidental orientation of the particles in the detection volume. In addition, no a priori differentiation between spherical and aspherical particles is possible for these instruments. To accurately determine the particle number concentration from the flow rate through the detection volume and the single particle count rate, careful calibration experiments have been performed using test aerosols with known number concentrations. Besides this calibration procedure, we are facing another difficulty to resolve the ice crystal number concentrations from the recorded data, namely the differentiation between the remaining seed aerosol particles and the nucleated ice crystals. This will be demonstrated in the following on the basis of selected measurements with the WELAS instrument.

The median diameter of the seed aerosol size distribution is typically about $0.3 \mu \mathrm{m}$ for the $\mathrm{H}_{2} \mathrm{SO}_{4} / \mathrm{H}_{2} \mathrm{O}$ solution droplets and $0.5 \mu \mathrm{m}$ for the mineral dust particles. Their refractive indices at visible wavelengths are about 1.4 and 1.5, respectively. Thus, a certain subset of the size distributions will extend into the measurement range of WELAS. As only a fraction of the seed aerosol particles is acting as ice nuclei during the expansion runs, the number concentration of un-activated seed aerosols has to be subtracted from the total WELAS count rate to precisely infer the actual ice parti- 
A

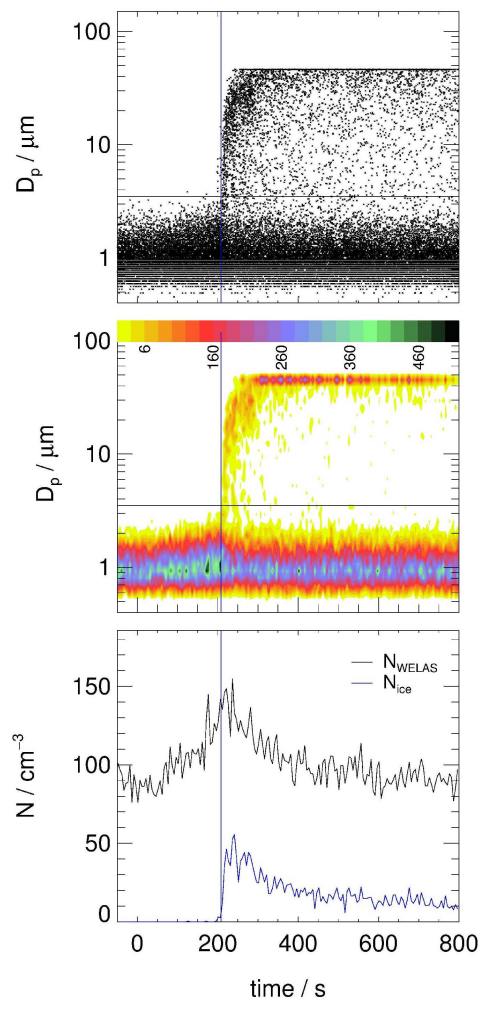

B
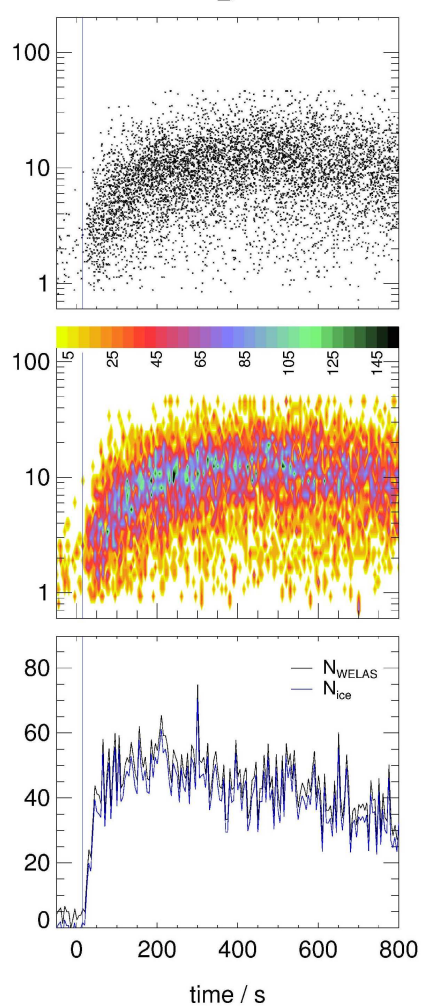

C
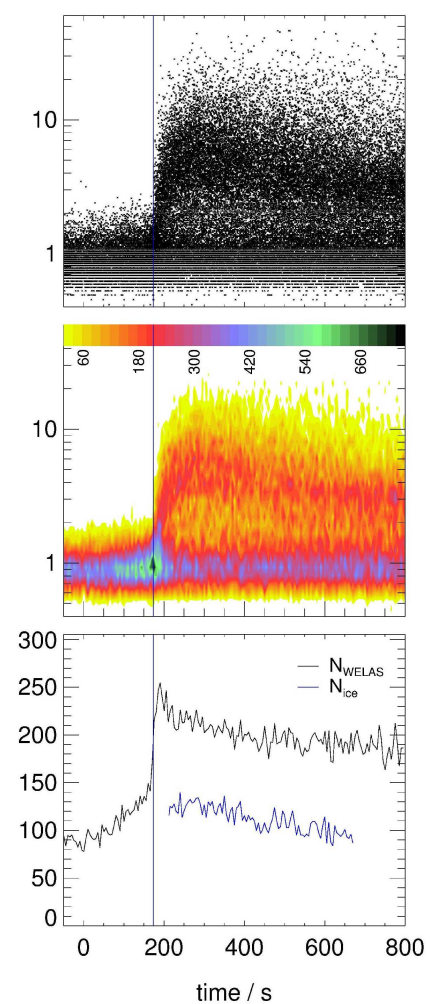

Fig. 2. WELAS recordings for three different AIDA expansion experiments, plotted as function of the experimental time. Left panel (A): Homogeneous freezing of supercooled $\mathrm{H}_{2} \mathrm{SO}_{4} / \mathrm{H}_{2} \mathrm{O}$ solution droplets at $224 \mathrm{~K}$. Middle panel (B): Heterogeneous ice nucleation on Saharan dust aerosol particles at $213 \mathrm{~K}$. Right panel (C): Homogeneous freezing of supercooled $\mathrm{H}_{2} \mathrm{SO}_{4} / \mathrm{H}_{2} \mathrm{O}$ solution droplets at $208 \mathrm{~K}$. Top row: Original WELAS scatter plots, each dot features a single particle count event in one of the WELAS size channels. Remember that for aspherical particles $D_{p}$ can only be interpreted as an "apparent" particle size. Middle row: Colour-coded size distributions, $\mathrm{d} N / \mathrm{dlog} D_{p}$ $\left(\mathrm{cm}^{-3}\right)$, obtained from the WELAS raw data using an integration time of $5 \mathrm{~s}$. Bottom row: Comparison between the overall particle number concentrations measured with WELAS, $N_{\text {WELAS }}(t)$, and the ice crystal number concentrations, $N_{\text {ice }}(t)$, which were deduced from $N_{\text {WELAS }}(t)$ using the strategies described in Sect. 2.2. Vertical lines in each panel denote the onset times of ice formation.

cle number concentration. The correction has to be carefully adjusted for each individual nucleation experiment. This is demonstrated in Fig. 2, showing three representative WELAS measurements where the degree of complexity of the subtraction procedure is gradually increasing. Panel (A) depicts an AIDA expansion run at $224 \mathrm{~K}$ with sulphuric acid droplets as seed aerosol, having a number concentration of about $2100 \mathrm{~cm}^{-3}$, as measured with a condensation particle counter (CPC 3010 , TSI). About $4 \%\left(90 \mathrm{~cm}^{-3}\right)$ of the $\mathrm{H}_{2} \mathrm{SO}_{4} / \mathrm{H}_{2} \mathrm{O}$ particles are detected by WELAS at time zero, denoting the start of pumping. Note that the WELAS counting efficiency for sulphuric acid droplets quickly approaches zero for particles smaller than about $0.9 \mu \mathrm{m}$, thereby feigning a maximum of the droplet size distributions at about this size. In the initial time period of pumping, the sulphuric acid solution droplets are growing by the uptake of water vapour as a result of the increasing relative humidity. Thereby, more droplets grow into the measurement range of WELAS, lead- ing to an enhanced count rate. After about $210 \mathrm{~s}$, the threshold relative humidity for homogeneous freezing is exceeded. The additional particle mode due to the nucleated ice crystals can be clearly identified in the single-particle scatter plot of the WELAS records. In this nucleation event, comparatively few ice particles are formed which then rapidly grow to large sizes, clearly beyond the size range of the remaining sulphuric acid solution droplets. Thus, separate counting of the ice crystals is possible by defining a threshold size (about $3.5 \mu \mathrm{m}$ in this example) and counting all particles above this threshold as ice particles.

In panel (B), an icing event due to deposition nucleation on Saharan dust aerosol particles at $213 \mathrm{~K}$ is shown, being typical for most of the AIDA expansion runs with mineral dust aerosols as ice nuclei. About $5 \mathrm{~cm}^{-3}$ of $80 \mathrm{~cm}^{-3}$ pre-added dust particles are seen by WELAS. Ice nucleation occurred soon after pumping was started, revealing the low critical ice saturation ratio for heterogeneous freezing. Mineral dust and 
ice particles are detected by the WELAS instrument in overlapping size ranges, so we cannot define a threshold to differentiate between them. However, mineral dust particles are very active ice nuclei, often yielding ice-activated fractions $f_{\text {ice }}$ greater than 0.5; see Table 2 in Möhler et al. (2006). For our study, we have purposely selected those expansion runs, where the number concentration of the nucleated ice crystals, $N_{\text {ice }}(t)$, rapidly exceeded $N_{\text {WELAS }}(t=0)$ by at least a factor of five, the latter denoting the number concentration of mineral dust particles counted by WELAS just before the expansion was started. As a first approximation, $N_{\text {ice }}(t)$ then roughly equals the WELAS number concentration measured at time $t$ during the ice nucleation event, $N_{\text {WELAS }}(t)$. More precisely, $N_{\text {ice }}(t)$ is obtained by subtracting the interfering dust particles from $N_{\text {WELAS }}(t)$ according to:

$$
\begin{aligned}
N_{\text {ice }}(t) & \cong N_{\text {WELAS }}(t) \\
& -N_{\text {WELAS }}(t=0) \frac{p}{p(t=0)}\left(1-f_{\text {ice }}(t)\right)
\end{aligned}
$$

In Eq. (1), $N_{\text {WELAS }}(t=0)$ is scaled by two correction factors. The first one accounts for dilution of the dust particles due to pumping, implying that no further dust particles are growing into the measurement range of WELAS by water uptake (Möhler et al., 2006). The second correction factor takes into consideration that a fraction $f_{\text {ice }}$ of the dust particles which are large enough to be detected by WELAS will also act as ice nuclei and therefore should not be subtracted from $N_{\text {WELAS }}(t)$. $f_{\text {ice }}(t)$ may be approximated by dividing $N_{\text {ice }}(t)$ through the total dust particle number concentration, $N_{\text {dust }}\left(t_{\text {ice }}\right)$, measured with the CPC immediately before the onset of the ice nucleation event at $t_{\text {ice }}$. This implies that there is no significant variation of the ice nucleating ability of the dust particles with particle size, i.e., that the fraction of larger dust particles which can be detected by WELAS is equally ice-active as the dust particle size distribution taken as a whole. Resolving Eq. (1) for $N_{\text {ice }}(t)$ then yields:

$N_{\text {ice }}(t) \cong \frac{N_{\text {WELAS }}(t)-N_{\text {WELAS }}(t=0) \frac{p}{p(t=0)}}{\left(1-\frac{N_{\text {WELAS }}(t=0)}{N_{\text {dust }}\left(t_{\text {ice }}\right)} \frac{p}{p(t=0)}\right)}$

Note that the second correction factor will usually affect $N_{\text {ice }}(t)$ by less than $10 \%$, given the criteria by which we have selected the expansion runs for our present study (i.e., $N_{\text {WELAS }}(t=0) \ll N_{\text {dust }}\left(t_{\text {ice }}\right)$ and $\left.N_{\text {ice }}(t)\right)$. Thus, the possible dependence of the ice nucleation efficiencies of solid particles on size, which was neglected in Eq. (2) but has been postulated on the basis of recent laboratory work (Archuleta et al., 2005), will be negligible. Particularly for the AIDA expansion runs with mineral dust particles, we have recorded numerous data sets with all three particle counting instruments in operation. A detailed inter-comparison of the performance of the various instruments has been reported by Möhler et al. (2006), showing that the results for the ice particle number concentrations usually differ by less than $\pm 20 \%$.
Panel (C) of Fig. 2 again shows a homogeneous freezing event of supercooled sulphuric acid droplets. The expansion run was started at a temperature of $208 \mathrm{~K}$ with an initial seed aerosol number concentration of $7200 \mathrm{~cm}^{-3}$. Compared to experiment $\mathrm{A}$, a larger number of smaller ice crystals is formed during pumping, implicating that the ice particle mode, although larger than the unfrozen solution droplets with sizes exceeding the detection threshold of WELAS, partially overlaps the droplet mode. Moreover, in contrast to experiment $\mathrm{B}$, the number concentration of the nucleated ice crystals is comparable to the number concentration of the seed aerosol particles that are visible to WELAS. Hence, none of the afore described strategies to derive $N_{\text {ice }}(t)$ can be applied. Instead of defining a threshold size for discriminating between sulphuric acid and ice particles as in experiment $\mathrm{A}$, we here employ a fitting procedure to subtract the $\mathrm{H}_{2} \mathrm{SO}_{4} / \mathrm{H}_{2} \mathrm{O}$ aerosol mode from the total WELAS count rate. We use a bimodal log-normal distribution function (see Eq. 5 in the next section) to fit the measured WELAS size distributions during the ice nucleation event, the first mode for representing the $\mathrm{H}_{2} \mathrm{SO}_{4} / \mathrm{H}_{2} \mathrm{O}$ droplets and the second mode for describing the ice particles. The fit was applied to size channels $>1.0 \mu \mathrm{m}$ with $100 \%$ counting efficiency. As to the sulphuric acid droplet mode, only the falling edge of the distribution at large particle diameters can be resolved by WELAS, suggesting that the accuracy of the fit result might be low. The values for the log-normal parameters of the $\mathrm{H}_{2} \mathrm{SO}_{4} / \mathrm{H}_{2} \mathrm{O}$ droplet mode, however, are constrained in several ways. Firstly, the droplet number concentration was provided from the independent CPC measurements. Secondly, the median droplet diameter before the start of the expansion was accurately measured with a differential mobility analyser (DMA), which operates at chamber conditions inside the thermostated housing of the AIDA facility; see Fig. 1. Model calculations can easily predict the change in droplet diameters as function of the increasing relative humidity during pumping (Haag et al., 2003). Thus only the mode width of the distribution remains as a variable parameter. Having retrieved its value from the bimodal fit, we can subtract the fraction of $\mathrm{H}_{2} \mathrm{SO}_{4} / \mathrm{H}_{2} \mathrm{O}$ particles which contribute to the overall WELAS count rate to deduce the number concentration of the ice crystals. Note that this bimodal fit will only produce reliable results when the median diameters of the sulphuric acid and the ice mode are distinctly separated. From the colour coded WELAS size distributions (middle row of panel C in Fig. 2) we can see that this criteria is met except from (i) the very beginning of the ice cloud formation at $t<220 \mathrm{~s}$, and (ii) the later part of the experiment at $t>650 \mathrm{~s}$ where the ice crystals are evaporating after the stop of pumping.

Besides the determination of the ice particle number concentration, another important quantity for the characterisation of ice clouds, namely the ice water content, can also be directly inferred from the AIDA measurements. Its value results from the difference of two humidity measurements. 

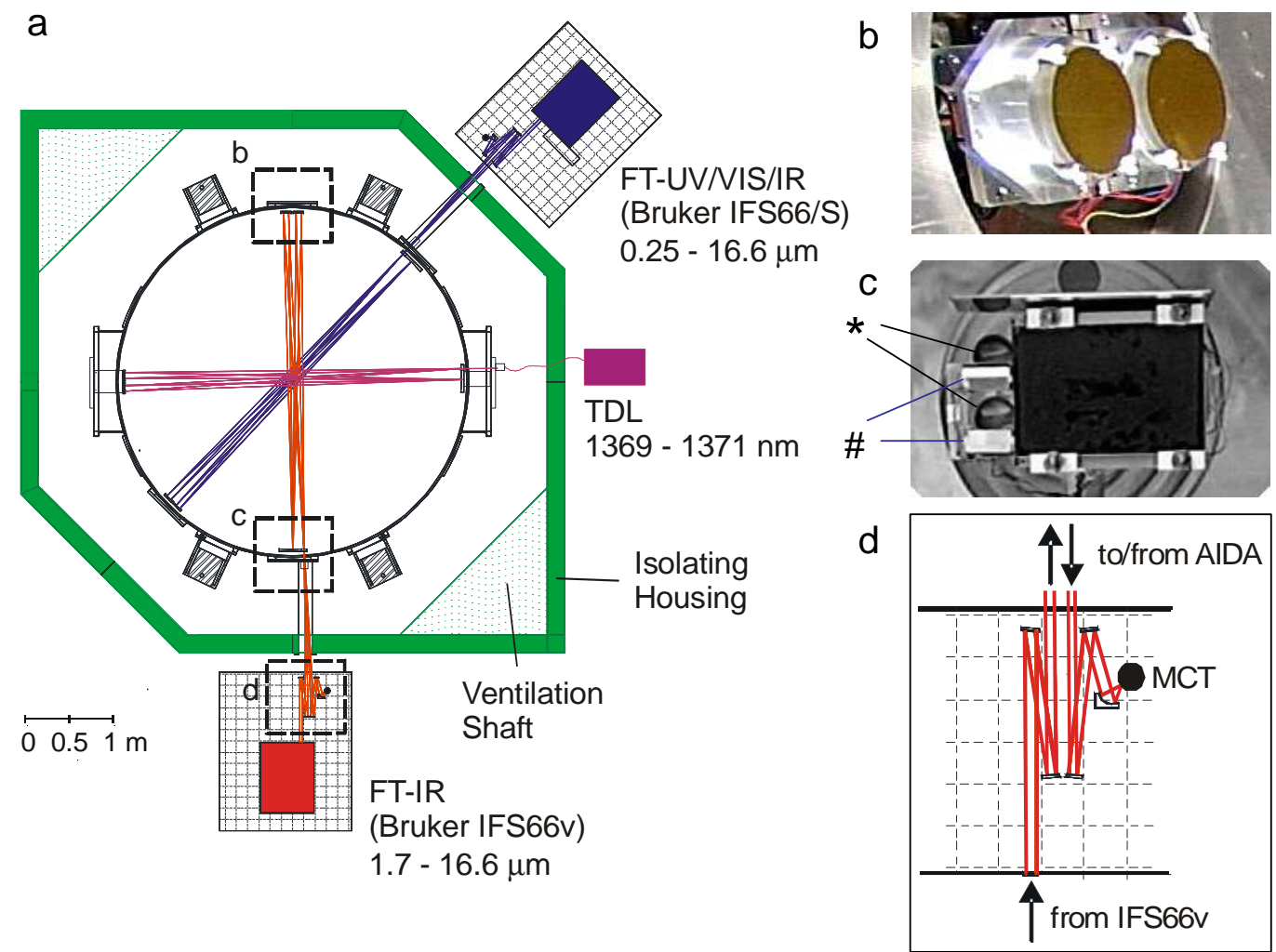

Fig. 3. (a) Scaled drawing of the AIDA cross-section at level 2. Three independent multiple reflection cells are used for in situ optical measurements, comprising FTIR extinction spectroscopy, TDL absorption spectroscopy for water vapour measurements, and extinction spectroscopy at visible wavelengths. Three enlarged sections show details of the set-up for the infrared measurements: (b) Picture of the gold-coated double mirrors of the White cell arrangement at the chamber walls opposite to the field mirror. The cabling belongs to the underlying heating foils. (c) Picture of the flange equipped with the mount of the field mirror which was demounted before taking this photo, the $\mathrm{BaF}_{2}$ entrance and exit windows (marked by *), and the pair of diagonal mirrors for doubling the number of cell traversals (marked by \#). (d) Sketch of the transfer optics for focussing the infrared beam into the chamber and onto the MCT detector.

Firstly, the total water mixing ratio, i.e., the sum of interstitial water vapour and evaporated ice crystal water, is measured with a fast scanning chilled mirror hygrometer (LX373, MBW), located outside the thermostated housing of the chamber (Fig. 1). In the heated $\left(30^{\circ} \mathrm{C}\right)$ stainless steel tube which is used as sampling line, all ice particles completely evaporate. Secondly, the interstitial gas phase water concentration in the presence of an ice cloud is measured by in situ tuneable diode laser (TDL) absorption spectroscopy at nearinfrared wavelengths (1368-1372 nm). Subtracting the TDL from the MBW data thus directly gives the $\mathrm{H}_{2} \mathrm{O}$ mass concentration of the ice crystals.

\subsection{FTIR extinction measurements and size distribution re- trieval}

The experimental arrangement for the infrared extinction measurements is shown in Fig. 3a, depicting a cross-sectional view of the AIDA cylinder at about $3.5 \mathrm{~m}$ height. The FTIR spectra are recorded in situ using an internal White-type mul- tiple reflection cell with a base length of $3.8 \mathrm{~m}$. The field mirror and its opposite double mirrors are mounted at the inside of two flanges which are enclosed in the chamber walls (see Figs. 3b-c). All mirrors are gold-coated and underlain with heating foils to prevent them from icing. The infrared beam exiting the FTIR spectrometer (type IFS 66v, Bruker) is directed into the White cell via two focussing mirrors (Fig. 3d), passing the chamber walls through a flange equipped with a heated $\mathrm{BaF}_{2}$ cell window (Fig. 3c). Note than in addition to the basic White type arrangement, a pair of diagonal mirrors (Fig. 3c) can be used to double the number of cell traversals by displacing the beam vertically and redirecting it to the double mirrors for another set of traversals (White, 1976). This set-up also minimises the influence of vibrations on the measurements. The beam exiting the White cell is collected using an identical set-up before it is focussed onto a liquidnitrogen cooled MCT detector (Fig. 3d). The extinction spectra are recorded from 6000 to 800 wavenumbers, confined by the transmittance of the $\mathrm{BaF}_{2}$ cell windows. To resolve the 


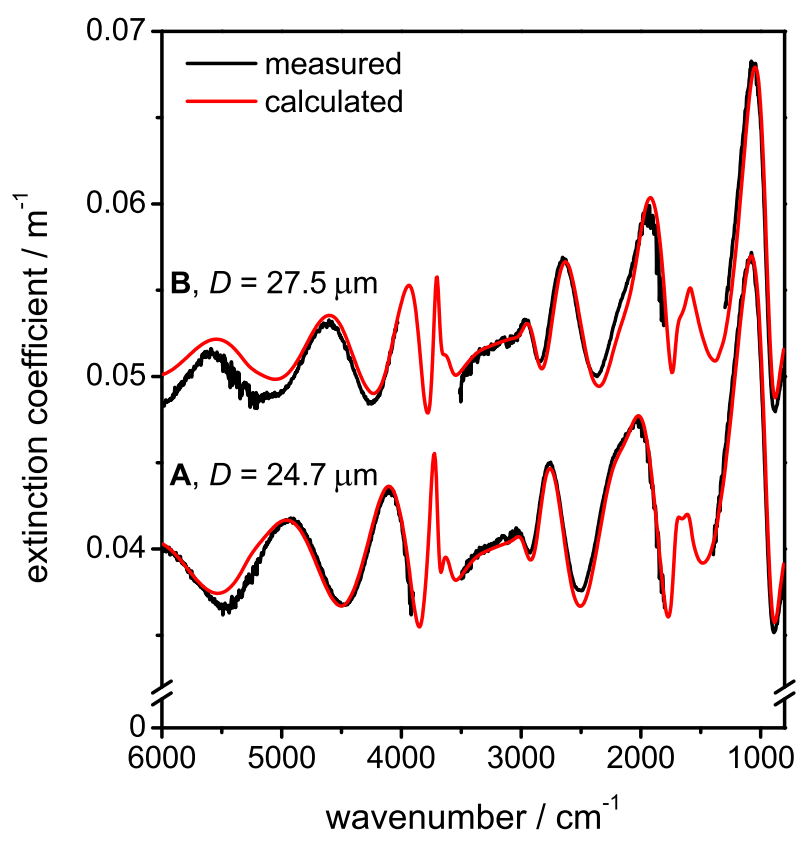

Fig. 4. Infrared extinction spectra of supercooled water droplets at $270 \mathrm{~K}$, recorded during an AIDA expansion run using Saharan dust aerosol particles as cloud condensation nuclei. The measured spectra (shown in black, regimes of strong water vapour absorption are omitted) are compared to the best fit results using Mie theory (red curves). Spectrum B is offset by $0.01 \mathrm{~m}^{-1}$ for clarity. For the Mie fits, a log-normal distribution function was employed (see Eq. 5 in Sect. 2.3). The retrieved droplet diameters are indicated in the figure, the mode width $\sigma_{g}$ of the size distribution was about 1.05 in both cases.

rapidly changing ice cloud properties during AIDA expansion cooling experiments, the infrared spectra were recorded at a rate of up to 6 spectra/min, typically by averaging over 40 scans at $4 \mathrm{~cm}^{-1}$ resolution.

The adjustment of the White cell mirrors is computercontrolled, using stepping motors especially designed for operation at the cold temperatures inside the isolating housing of the chamber. The alignment is done by switching to the halogen light source of the spectrometer. Small video cameras are used to observe the spots on the mirrors for controlling the optical path length. As the extinction coefficients of the ice clouds which are generated during the AIDA expansion runs strongly vary with the initial gas temperature, i.e., with the amount of water vapour available for ice crystal growth, the optical path length is individually adjusted for each ice nucleation experiment. Typically, optical paths ranging from $23 \mathrm{~m}$ ( 6 traversals) to $257 \mathrm{~m}$ (68 traversals) are used, thereby, on the one hand, avoiding saturation of the infrared bands at the higher temperatures and, on the other hand, increasing the sensitivity at the lowest temperatures.

Due to its finite field of view, some forward-scattered light may be collected by the detector, thereby resulting in an underestimation of the true extinction (Bohren and Huffman, 1983). As the fractional error in the extinction measurements generally increases with increasing size parameter $x=\pi D_{p} / \lambda$ (Arnott et al., 1995), the infrared spectra of the super- $\mu \mathrm{m}$ sized ice crystals formed in the expansion experiments might be biased, especially at higher wavenumbers. Based on previous theoretical studies on the forward scattering correction factors (Deepak and Box, 1978; Moosmüller and Arnott, 2003; Wind and Szymanski, 2002), however, the percentage error for the set-up of our transmission experiment is estimated to be below 5\% even for size parameters as large as 50, corresponding to a sphere diameter of $25 \mu \mathrm{m}$ at $6000 \mathrm{~cm}^{-1}$. In general, the apparent change of extinction due to the forward-scattering contribution, much in the same way as an incorrect assumption on particle shape (see Sect. 1), would also lead to distortions in the retrieved particle size distribution (Wind and Szymanski, 2002). But if, in return, the size distribution of an aerosol would be known with high precision, the comparison between measured and calculated, i.e., true extinction would directly point to the fractional error owing to forward-scattering. By chance, a specific AIDA expansion run allows us to carry out this comparison in order to confirm our error estimation. In this particular experiment, using mineral dust particles as seed aerosol at an initial temperature of $270 \mathrm{~K}$, a cloud consisting exclusively of supercooled water droplets was generated after exceeding $100 \%$ relative humidity with respect to supercooled water during the expansion; see e.g. Fig. 3 in Wagner et al. (2005a). The infrared spectra of the water cloud bare a pronounced Mie interference structure at non-absorbing wavelengths (Bohren and Huffman, 1983) as the nuclei all grow to droplets of roughly the same diameter, i.e., the AIDA chamber just operates as a condensation nuclei counter in this type of experiment. As the oscillations in the interference structure are very sensitive to the droplet diameter, the size distribution of the water cloud can be retrieved with high accuracy, using reliable infrared optical constants for supercooled water (Wagner et al., 2005a; Zasetsky et al., 2005). Figure 4 shows two measured infrared extinction spectra of the droplet cloud, recorded at different times during the expansion, in comparison with the best fit results from Mie calculations. For spectrum A, a droplet diameter of $24.7 \mu \mathrm{m}$ was retrieved. Note the excellent agreement between measured and calculated spectrum over the full wavelength regime, covering a range of size parameters from $x=6$ at $800 \mathrm{~cm}^{-1}$ to $x=46$ at $6000 \mathrm{~cm}^{-1}$. Thus, even towards higher wavenumbers, no underestimation of extinction due to forward-scattering can be observed. The forward-scattering contribution only becomes visible for even larger droplet diameters, i.e., in spectrum B with $D=27.5 \mu \mathrm{m}$. Here, the measured infrared spectrum shows a clear tendency of underrating the true optical depth at the highest wavenumbers. The relative error, however, still does not exceed $4 \%$. As the ice crystals, whose infrared spectra will be analysed in this study, are of much smaller sizes (1 to 15 microns), the forward-scattering correction, regardless 
of its dependence on particle shape (Bohren and Koh, 1985), will be negligible.

AIDA expansion runs which resulted in the formation of clouds of supercooled water droplets also prove valuable to check whether it is in principle justified to directly compare size distributions of micron-sized cloud particles retrieved from the FTIR spectra to independent OPC measurements, given that the location of these two probes is fundamentally different. Whereas the infrared extinction spectra are recorded in situ at medium height of the AIDA vessel, the OPCs operate in an ex situ mode by sampling chamber air from a distant point near the bottom of the chamber. In the case of spherical droplets, sizing by the OPCs will be correct and the comparison with the FTIR retrieval results with respect to the cloud droplet diameters will reveal whether both techniques are actually sizing particles of the same size. We have always observed good agreement between the cloud droplet diameters deduced from the FTIR retrievals and the OPC measurements; see e.g. Fig. 5 in Benz et al. (2005) and Fig. 13 in Wagner et al. (2005a). This illustrates that the operation of the mixing fan (see Fig. 1) provides for sufficiently homogeneous cloud properties throughout the chamber volume.

The basic procedure for the quantitative analysis of the extinction spectra of the ice crystals has been adopted from previous laboratory studies on the characterisation of aerosols from infrared optical depth measurements (Arnott et al., 1997b; Liu et al., 1999; Zasetsky et al., 2004). It is based on the Lambert Beer equation, relating the measured optical depth $\tau\left(\tilde{v}_{j}\right)$ at a specific wavenumber $\tilde{v}_{j}$ according to

$$
\begin{gathered}
\tau\left(\tilde{v}_{j}\right)=-\log \frac{I\left(\tilde{v}_{j}\right)}{I_{0}\left(\tilde{v}_{j}\right)}=\frac{L}{\ln 10} \underbrace{\sum_{i=1}^{N} n\left(D_{i}\right) C_{\text {ext }}\left(D_{i}, \tilde{v}_{j}\right)}_{\text {extinction coefficient }} \\
j=1 \ldots M
\end{gathered}
$$

with the optical path length $L$, the number concentration $n\left(D_{i}\right)$ of particles of size $D_{i}$, and the extinction cross section $C_{\text {ext }}\left(D_{i}, \tilde{v}_{j}\right)$. We note at this point that the term "diameter $D$ ", when referring to ice crystals, is meant to denote the equal-volume sphere diameter throughout this paper. Thus, a $M \times N$ matrix of extinction cross sections, with $M$ denoting the number of wavenumber grid points in the spectrum and $N$ the number of discrete diameters in the considered particle size range, has to be calculated as input for the retrieval algorithm. The size distribution of the ice crystals may then be retrieved by minimising the summed squared residuals between measured and calculated spectra, using the individual $n\left(D_{i}\right)$ as fitting parameters. Note that, in contrast to the OPCs, the spectral analysis of the ice clouds is not hindered by the seed aerosol particles as their volume concentration is usually negligible compared to the large ice volume concentrations which evolve during the expansion runs. After having defined the general framework for the retrieval, three important questions have to be considered before its actual implementation: (i) Which concept is used to account for the aspherical habits of the ice crystals? (ii) Which refractive indices are employed to calculate the infrared extinction cross sections? (iii) Is it reasonable to impose any constraints on the retrieved size distribution, e.g. by using a smoothing algorithm or even employing a particular distribution function? These issues will be briefly addressed in the following.

For our study, we have chosen to model the aspherical ice particles as finite circular cylinders. The extinction cross sections were calculated with the extended precision FORTRAN T-matrix code for randomly oriented particles by Mishchenko and Travis (1998). Five different aspect ratios $\phi$, i.e., the ratios of the diameter to the length of the cylinders, were selected to assess the influence of particle shape on the retrieval results, namely $\phi=0.5,0.7,1.0,2.0$, and 3.0. For each aspect ratio, a $76 \times 167$ matrix of extinction cross sections was calculated, i.e.: 167 discrete diameters, specified in terms of the equal-volume sphere diameter, from 0.1 to $25.0 \mu \mathrm{m}$ at equidistant $0.15 \mu \mathrm{m}$ size steps, as well as 76 equidistant wavenumber elements from 4500 to $800 \mathrm{~cm}^{-1}$. Only for the two most compact shapes $\phi=0.7$ and 1.0, the range of computable wavenumbers could be extended up to $6000 \mathrm{~cm}^{-1}$, being the upper limit of our measurements. Otherwise, the T-matrix computations for $\tilde{v}>4500 \mathrm{~cm}^{-1}$ did not converge for the entire range of particle diameters. For $\phi<0.5$ and $\phi>3.0$, convergence problems also appeared in the regime of the $\mathrm{O}-\mathrm{H}$ stretching mode, especially at wavenumbers around $3200 \mathrm{~cm}^{-1}$, where both the real and imaginary parts of the refractive index of ice are increasing, thereby reducing the maximal convergent size parameters in the T-matrix calculations; see Table 3 in Mishchenko and Travis (1998). Note that for a given size parameter, more extreme aspect ratios can be computed for plate-like cylinders $(\phi>1)$ than for columns $(\phi<1)$.

Lee et al. (2003) have shown that for the calculation of extinction efficiencies at infrared wavelengths $(8-12 \mu \mathrm{m})$, circular cylinders are a reasonable surrogate for pristine hexagonal ice crystals. Hexagonal columns and plates, however, are only two representatives from the broad variety of crystal shapes, although these basic hexagonal structures might be the dominant fraction for small ice crystals in high-altitude cirrus clouds (Eremenko et al., 2005). Thus, in most cases, the ice clouds generated during the AIDA expansion experiments will consist of an ensemble of differently shaped aspherical particles. To account for this in the retrieval strategy, we may employ a shape distribution of cylinders to calculate the infrared extinction cross sections. This methodology has already been applied in numerous studies to model light scattering by aspherical particles (Baran, 2003; Hill et al., 1984; Mishchenko, 1993). By defining a shape-averaged extinction cross section $\left\langle C_{\text {ext }}\right\rangle\left(D_{i}, \tilde{v}_{j}\right)$ as follows, 


$$
\begin{aligned}
\left\langle C_{\mathrm{ext}}\right\rangle\left(D_{i}, \tilde{v}_{j}\right) & =a_{1} \cdot C_{\mathrm{ext}}^{\phi=0.5}\left(D_{i}, \tilde{v}_{j}\right)+a_{2} \cdot C_{\mathrm{ext}}^{\phi=0.7}\left(D_{i}, \tilde{v}_{j}\right) \\
& +a_{3} \cdot C_{\mathrm{ext}}^{\phi=1.0}\left(D_{i}, \tilde{v}_{j}\right)+a_{4} \cdot C_{\mathrm{ext}}^{\phi=2.0}\left(D_{i}, \tilde{v}_{j}\right) \\
& +a_{5} \cdot C_{\mathrm{ext}}^{\phi=3.0}\left(D_{i}, \tilde{v}_{j}\right)
\end{aligned}
$$

with $\sum_{i} a_{i}=1$, we can analyse the influence of different combinations of the mixing factors $a_{i}$ on the retrieval results. Therefore, the individual $a_{i}$ were varied on a $20 \%$ grid (i.e., $a_{1}=1, a_{2-5}=0 / a_{1}=0.8, a_{2}=0.2$, $a_{3-5}=0 / a_{1}=0.6, a_{2}=0.4, \quad a_{3-5}=0 / a_{1}=0.6, a_{2}=0.2, a_{3}=0.2$, $\left.a_{4-5}=0 / \ldots / a_{1-5}=0.2 / \ldots / a_{1-4}=0, a_{5}=1\right)$, altogether summing up to 126 permutations of the mixing coefficients. For each set of $a_{i}$, the size distribution of the ice crystals was retrieved from the measured infrared spectra, thereby illustrating the spreading of the fit results over the range of considered particle habits, comprising both pure cylindrical shapes and shape distributions of cylinders with different aspect ratios.

As input for our T-matrix calculations in the 3700 $800 \mathrm{~cm}^{-1}$ range, we employed the low-temperature refractive indices for ice from the study by Clapp et al. (1995). The authors have demonstrated the pronounced temperature dependence of the optical constants, particularly in the regime of the $\mathrm{O}-\mathrm{H}$ stretching mode at about $3250 \mathrm{~cm}^{-1}$. Imprecise refractive indices, in much the same way as erroneous assumptions on particle shape, may also lead to strong distortions of the retrieved size distributions, especially towards smaller particles. This was clearly shown by Liu et al. (1999), considering as example three slightly different refractive index data sets for liquid water (see Figs. 8 and 9 therein). For our calculations, we therefore used the Clapp et al. (1995) data set determined at $210 \mathrm{~K}$, being in the middle of the temperature range of our measurements. For wavenumbers $>3700 \mathrm{~cm}^{-1}$, the near-infrared optical constants of water ice from Rajaram et al. (2001) were appended to the Clapp et al. (1995) data set. Last year, Zasetsky et al. (2005) have published further low-temperature complex refractive index data sets for ice, determined from aerosol extinction spectra using an approach similar to the earlier Clapp et al. (1995) study. The frequency range of these measurements, however, was extended to $460 \mathrm{~cm}^{-1}$, thus fully covering the intense intermolecular rotational mode of ice centred at about $800 \mathrm{~cm}^{-1}$. The incorporation of this low-frequency absorption band in the Kramers-Kronig integration to calculate the spectrum of the real part $n$ of the refractive index from its imaginary part $k$ improves the accuracy of the $n$ spectrum at wavenumbers from $1500-800 \mathrm{~cm}^{-1}$ (Zasetsky et al., 2005). For that reason, we repeated the $\mathbf{T}$-matrix calculations in this frequency regime with the novel Zasetsky et al. (2005) data set.

The most unbiased approach to retrieve number size distributions of ice crystals from measured infrared spectra would consist in an independent variation of all 167 number concentration elements $n\left(D_{i}\right)$, only constrained by applying a smoothing algorithm to avoid unphysical sharp features in the deduced size distribution. Arnott et al. (1997b) for in(4) stance have described an easy-to-implement iterative algorithm for that purpose which we have adopted and tested for our present study. But we know from the study by Liu et al. (1999) that even the slightest inaccuracies of the employed refractive indices, or an approximation of the actual particle shape, which is inevitable in any realistic retrieval problem, will deform the real size distribution. In particular, a pseudo mode of small particles might emerge, thereby repairing the mismatch between measured and calculated spectra induced by the imprecision of the optical constants and/or the particle shape representation. This additional small particle mode will not interfere with the accurate retrieval of the ice volume densities but will rule out the precise determination of the ice particle number concentrations, being the central objective of the AIDA ice nucleation experiments. We therefore chose to use the analytical lognormal distribution function $\Phi\left(D_{p}\right)$ to describe the number size distribution of the ice crystals,

$$
\Phi\left(D_{P}\right)=\frac{1}{\sqrt{2 \pi} D_{p} \ln \sigma_{g}} \exp \left\{-\frac{\left(\ln D_{p}-\ln D\right)^{2}}{2\left(\ln \sigma_{g}\right)^{2}}\right\}
$$

where $\Phi\left(D_{p}\right) d D_{p}$ denotes the fraction of ice particles per unit volume having particle sizes between $D_{p}$ and $D_{p}+d D_{p}$. The ice particle number concentration $N$ as well as the count median diameter $D$ and the mode width $\sigma_{g}$ of the $\log$ normal size distribution were then retrieved by minimising the summed squared residuals between measured and calculated spectra with the downhill simplex algorithm as the optimisation technique (Press et al., 1992). We have also tested the modified gamma distribution function (Mishchenko and Travis, 1998), but we usually retrieved shape parameters producing a distribution function which closely mimicked the log-normal type.

For the majority of the AIDA expansion runs discussed in this paper, it is appropriate to use a unimodal distribution function. In the case of homogeneous freezing of supercooled $\mathrm{H}_{2} \mathrm{SO}_{4} / \mathrm{H}_{2} \mathrm{O}$ solution droplets, relative humidity with respect to ice rapidly decreases after the onset of ice nucleation due to the fast depositional growth of the generated ice crystals; see Fig. 4 in Haag et al. (2003). After falling below the critical threshold relative humidity, no further solution droplets can freeze. Within this short timeframe, only $\mathrm{H}_{2} \mathrm{SO}_{4} / \mathrm{H}_{2} \mathrm{O}$ droplets in a narrow range of the overall size distribution can freeze, leading to a unimodal distribution of pristine ice crystals. The mode width of this distribution is then gradually narrowing during the growth of the ice crystals by water vapour deposition; see Fig. 8 in Haag et al. (2003). As to the expansion runs with mineral dust particles as ice nuclei, we have observed that the formation of new ice particles is also limited to a short time period after exceeding the critical ice saturation ratio for deposition ice nucleation (Möhler et al., 2006). For most of the expansion experiments, the OPCs have detected a sin- 
gle nucleation event, leading to a single-mode distribution of ice particles. Discrimination between single and dual nucleation modes can be clearly discerned in the scatter plots of the particle counting instruments (see Fig. 2). Only occasionally, a second nucleation event at higher ice saturation ratios could be observed, see e.g. panel (C) of Fig. 1 in Field et al. (2006), mostly in the third or forth repetitive expansion run with the same desert dust sample. Apparently, after the most active dust particles had been removed by sedimentation of large ice crystals during the first two expansion runs, a second nucleation mode, attributed to a subset of less active particles, was detected. Considering the complexity of the size distribution retrieval for aspherical particles, we will at first focus on the expansion runs with single ice nucleation modes. Afterwards, we will present one selected case study of a dual nucleation event to explore whether it is also possible to accurately retrieve a more complicated bimodal size distribution of ice crystals from infrared extinction spectra. For this purpose, we have analysed an AIDA expansion run especially designed to provoke two nucleation modes. Here, an external mixture of supercooled sulphuric acid solution droplets and dry mineral dust particles, the latter ones in small number concentration, was injected into the AIDA chamber. The first ice nucleation event was triggered by the mineral dust particles owing to their low threshold relative humidity compared to homogeneously freezing sulphuric acid droplets. But because of the small number concentration of the heterogeneously nucleated ice crystals and the relatively high pumping speed, the critical ice saturation ratio for homogeneous freezing of the $\mathrm{H}_{2} \mathrm{SO}_{4} / \mathrm{H}_{2} \mathrm{O}$ solution droplets was also exceeded somewhat later during the expansion, leading to a second ice nucleation event.

\section{Exemplary results of the T-matrix calculations}

Selected results of our T-matrix computations are shown in Fig. 5, illustrating plots of the extinction cross sections and extinction efficiencies $\left(Q_{\text {ext }}\right)$ of the ice crystals as a function of particle size for three different wavenumbers: (i) $4456.8 \mathrm{~cm}^{-1}$ (non-absorbing wavenumber at the highfrequency end of our infrared spectra, corresponding values for the optical constants: $n=1.26$ and $k<10^{-3}$ ), (ii) $3251.4 \mathrm{~cm}^{-1}$ (centre of the intense $\mathrm{O}-\mathrm{H}$ stretching mode with $n=1.22$ and $k=0.78$ ), and (iii) $1226.5 \mathrm{~cm}^{-1}$ (regime of little absorption at lower wavenumbers with $n=1.32$ and $k=0.04$ ). Each graph contains the results for the five purely cylindrical shapes as well as for one selected distribution of cylindrical shapes, setting all mixing factors $a_{i}$ equal to 0.2. For comparison, Mie calculations for spherical particles are also shown.

At absorbing wavenumbers $\left(k>0.05\right.$, e.g. at $\left.3251.4 \mathrm{~cm}^{-1}\right)$, the extinction cross sections smoothly increase over the full range of particle sizes. The deviations between the results for the various shapes are not severe, as will be shown by comparing the computations for the most compact $(\phi=1)$ and the most aspherical $(\phi=3)$ cylindrical shapes. At an equal-volume sphere diameter of $5.5 \mu \mathrm{m}$, for example, the extinction cross section for $\phi=3$ is enhanced by about $10 \%$ in comparison with $\phi=1$. This percentage difference only slightly increases with increasing particle size (e.g., $11.5 \%$ for $15 \mu \mathrm{m}$-sized ice crystals). At non-absorbing wavenumbers or regimes with low absorption $(k<0.05$, e.g. at 4456.8 and $1226.5 \mathrm{~cm}^{-1}$ ), the extinction plots are governed by the characteristic interference structure, i.e., series of extinction maxima and minima caused by interference of incident and forward-scattered light (Bohren and Huffman, 1983). Only for particle sizes below the first interference maximum (about $6 \mu \mathrm{m}$ for $4456.8 \mathrm{~cm}^{-1}$ and $15 \mu \mathrm{m}$ for $1226.5 \mathrm{~cm}^{-1}$ ), the extinction cross sections for the variously shaped ice particles show little variation. In this regime, the extinction cross sections (respectively scattering cross sections in the case of no absorption) are slightly decreasing with increasing degree of asphericity; see expanded graph in the top left panel of Fig. 5. The percentage deviation between the results for $\phi=1$ and $\phi=3$, however, always remains below $10 \%$. In contrast, extinction dramatically varies with particle shape for diameters larger than the first interference maximum. With increasing asphericity, the amplitude of the oscillations in the extinction efficiencies is successively damped, accompanied by a shift in the positions of the extinction maxima and minima (Hill et al., 1984; Proctor and Harris, 1974; Yang et al., 2001). For $\phi=3$, very little regular interference structure can be discerned. Applying the shape distribution of ice cylinders also strongly dampens the oscillations, except from the first extinction maximum. The extinction cross sections for the different particle shapes can vary by more than $50 \%$, e.g. at a diameter of about $18 \mu \mathrm{m}$ for $4456.8 \mathrm{~cm}^{-1}$, representing an extinction minimum for compact cylinders $(\phi=1)$ but extinction maxima for $\phi=0.5$ or $\phi=2.0$.

Note that, particularly within the regime of the interference structure, the changes in the extinction efficiencies for cylindrical ice crystals due to varying aspect ratios are much larger than the relative errors introduced by approximating hexagonal ice crystals with equivalent circular cylinders (Lee et al., 2003). Lee et al. (2003) have compared the extinction efficiencies for hexagonal columns and various equivalent circular cylinders, both at a strongly absorbing $(11.0 \mu \mathrm{m}$, $\left.909 \mathrm{~cm}^{-1}, k=0.3\right)$ and a weakly absorbing infrared wavelength $\left(8.5 \mu \mathrm{m}, 1176 \mathrm{~cm}^{-1}, k=0.04\right)$; see Figs. 3 and 6 in their paper. The relative error in $Q_{\text {ext }}$ when approximating the ice hexagons with e.g. equivalent volume circular cylinders of the same aspect ratio as the hexagonal columns was clearly below $10 \%$ for the complete considered particle size range. In particular, the habitus of the interference structure in the $Q_{\text {ext }}$ plot at the weakly absorbing $8.5 \mu \mathrm{m}$ wavelength for ice hexagons was also accurately reproduced by the calculations for equivalent circular ice cylinders. We therefore consider it justified to have employed the simpler circular cylinder geometry in our calculations. 

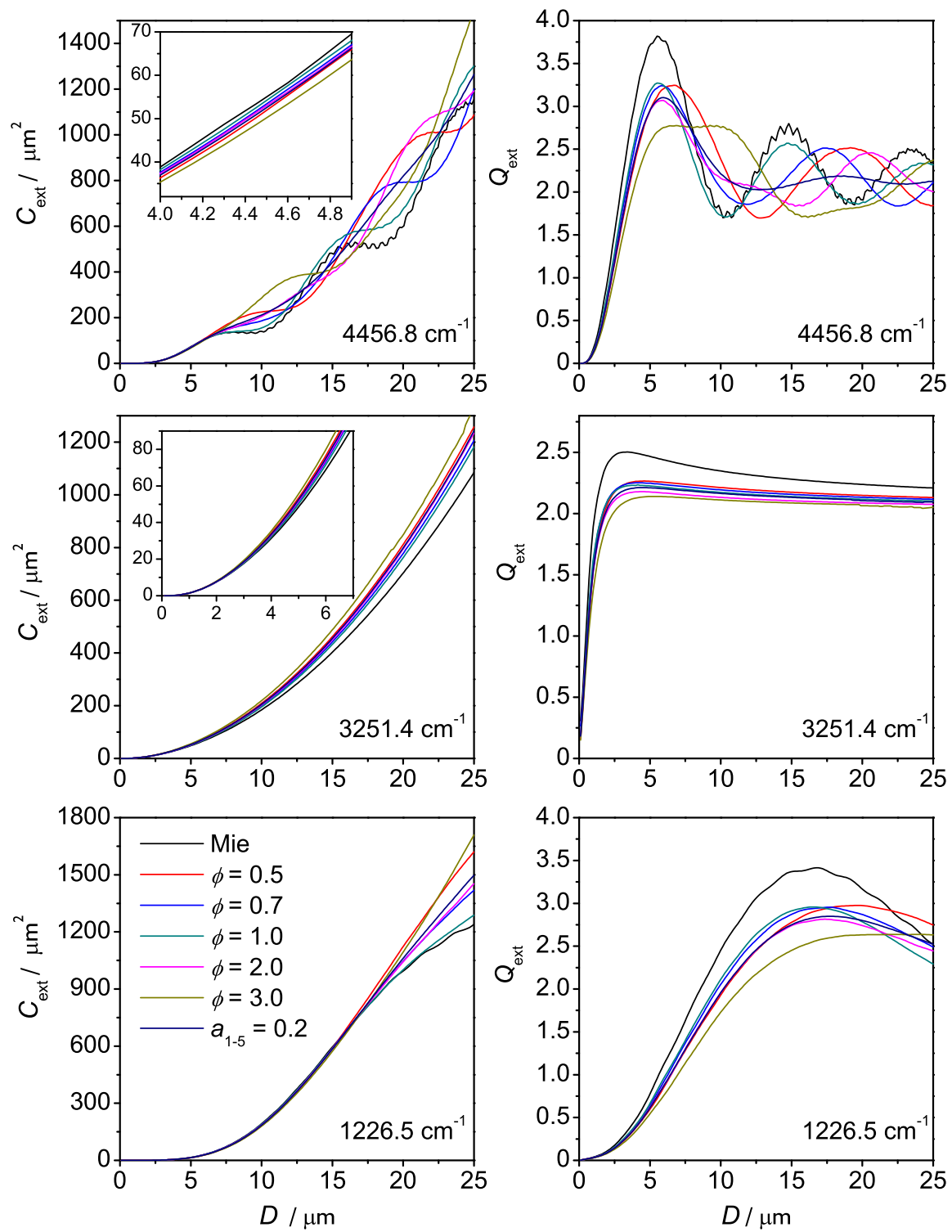

Fig. 5. Extinction cross sections $C_{\text {ext }}$ (left column) and extinction efficiencies $Q_{\text {ext }}$ (extinction cross sections divided through the crosssectional areas of the particles, projected on a plane perpendicular to the incident beam, right column) as a function of the equal-volume sphere diameter $D$ for differently shaped ice crystals at three wavenumbers, calculated with the T-matrix code for randomly oriented particles. The colour coding is shown in the bottom left panel. In each graph, the results for five purely cylindrical shapes with different aspect ratios are compared to the results for a distribution of cylindrical shapes and for spherical ice crystals from Mie theory.

When considering potential implications of the T-matrix results shown in Fig. 5 for the size distribution retrieval procedure, it is useful to discuss two different scenarios: (i) The main part of the size distribution of the nucleated ice crystals extends below the first interference maximum, and (ii) the ice crystals grow into the range of particle sizes which give rise to distinct oscillations of the extinction cross sections and efficiencies at non-absorbing wavenumbers. Concerning the first case, ice crystal diameters below about $6 \mu \mathrm{m}$ would assure that for the complete wavenumber range of our measurements, respectively $\mathbf{T}$-matrix calculations, the first interference maximum is not reached. Consequently, at all wavenumbers, either absorbing or non-absorbing, the extinction cross sections will smoothly increase with particle size and the deviations for the different considered particle shapes will not exceed $10 \%$. As a result, each shape assumption, even approximating the aspherical ice crystals by spheres, will inevitably lead to a close agreement between measured and calculated infrared extinction spectra for this range of crystal sizes, aptly referred to as "unreasonable effectiveness 
Table 1. Retrieved parameters of the log-normal size distribution when fitting a reference infrared spectrum of cylindrical ice crystals of aspect ratio $\phi=1$ with different assumptions on particle shape. The deduced ice volume concentrations $V$ are also shown.

\begin{tabular}{lcccc}
\hline Crystal Shape & $N / \mathrm{cm}^{-3}$ & $\sigma_{g}$ & $D / \mu \mathrm{m}$ & $V / \mu \mathrm{m}^{3} / \mathrm{cm}^{3}$ \\
\hline$\phi=\mathbf{1 . 0}$ & $\mathbf{1 0 0 . 0}$ & $\mathbf{1 . 3 0}$ & $\mathbf{4 . 0 0}$ & $\mathbf{4 5 6 8}$ \\
$\phi=0.5$ & 73.9 & 1.25 & 4.58 & 4678 \\
$\phi=0.7$ & 89.2 & 1.28 & 4.22 & 4589 \\
$\phi=2.0$ & 82.9 & 1.24 & 4.39 & 4552 \\
$\phi=3.0$ & 59.1 & 1.10 & 5.24 & 4635 \\
$a_{1-5}=0.2$ & 78.3 & 1.23 & 4.52 & 4556 \\
sphere (Mie) & 114.5 & 1.35 & 3.72 & 4592 \\
\hline
\end{tabular}

of mimicking measured infrared extinction by hexagonal ice crystals with Mie ice spheres" in the literature (Arnott et al., 1997a). Only, the deviations in extinction for the various shapes will be converted into differences of the retrieval results for the size distribution of the ice crystals. To exemplify the magnitude of this solution ambiguity, we have calculated the extinction spectrum for log-normally distributed cylindrical ice crystals with $\phi=1$ and thereafter retrieved the size distribution for $\phi=0.5,0.7,2.0$, and 3.0 as well as for the shape distribution with all $a_{i}=0.2$. For comparison, Mie theory was also applied in the retrieval. $N=10 \mathrm{~cm}^{-3}, \sigma_{g}=1.3$, and $D=4 \mu \mathrm{m}$ were chosen as input parameters of the size distribution for the $\phi=1$ spectrum. Figure 6 shows the extinction coefficients for $\phi=1$ together with the best fit results for $\phi=0.5,2.0,3.0$, and Mie theory. Note the good agreement between all spectra except from the slight mismatch near the extinction maximum at $2900 \mathrm{~cm}^{-1}$. As indicated above, this mismatch can be "repaired" to a certain extent by adding spurious modes of smaller and/or larger ice crystals when relaxing the log-normal constraint on the size distribution retrieval. The various retrieval results, implying the log-normal distribution function, are summarised in Table 1. Note that the fit result using Mie theory systematically deviates from the outcomes for every single one of the cylindrical shapes, underlining that it is not appropriate to justify its use by arguing that averaging over different particle habits could remove the sensitivity of the retrieval results to the particle shape. For the comparatively compact cylindrical shapes $\phi=0.5,0.7$, 1.0 , and 2.0 as well as the shape distribution, the spreading in the retrieved particle sizes is quite limited, ranging from $4.00-4.58 \mu \mathrm{m}$. The more aspherical the particle shape, the larger are the retrieved ice crystal median sizes. This trend can be explained as follows. With increasing asphericity, larger crystal sizes are needed to accurately reproduce the infrared spectrum for $\phi=1$ at non-absorbing wavenumbers $>3600 \mathrm{~cm}^{-1}$ as a result of the decreasing scattering cross sections. In order to precisely match the $\phi=1$ spectrum also at absorbing wavenumbers, being sensitive to the

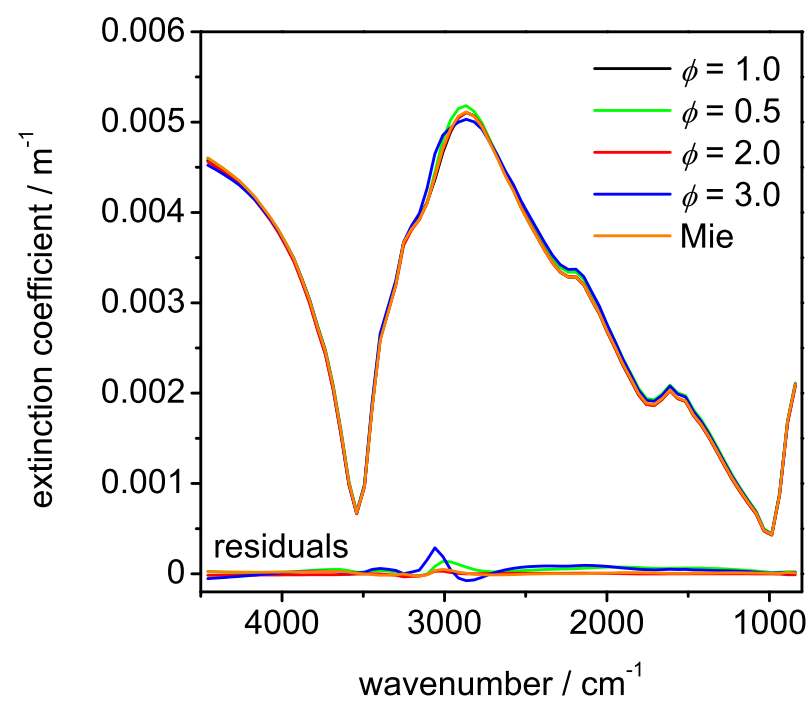

Fig. 6. Reference extinction spectrum of log-normally distributed cylindrical ice crystals with $\phi=1\left(N=10 \mathrm{~cm}^{-3}, \sigma_{g}=1.3\right.$, and $D=4 \mu \mathrm{m}$ ) in comparison with the best fit results for different shape assumptions. The spectral residuals (differences to the $\phi=1$ spectrum) are shown in the bottom part. The retrieved size distributions for the various particle habits are summarised in Table 1.

volume concentration of ice, the retrieved number concentrations of the ice crystals must consequently decrease with increasing degree of asphericity. Note that the retrieved volume concentrations of ice are virtually unaffected by these distortions in the size distributions, the percentage deviations being less than 3\%. The relative scatter in the retrieved ice particle number concentrations is larger compared to the crystal sizes because the latter contribute to the particle volume concentration by the power of three. For the compact shape parameters, the deduced number concentrations are ranging from $74-100 \mathrm{~cm}^{-3}$; for crystals with the largest aspect ratio $(\phi=3.0)$, the fit result shows even stronger variation. All the same, for the variety of considered particle shapes, the extent of this solution ambiguity of the FTIR retrievals is comparable to the precision by which the ice particle number concentrations can be measured with the OPCs $( \pm 20 \%)$. Consequently, it is hardly possible to infer the actual shape of the ice crystals from the infrared spectra by comparing the retrieval results with data from the OPCs. Only if the spectrum of the retrieved number concentrations would clearly lie outside the bandwidth of the OPC data, and any instrumental problems for the latter could be ruled out, this could point to the presence of an ice cloud composed of crystals with extreme aspect ratios (needle-like or plate-like) whose scattering properties strongly differ from those of moderately aspherical particles (Zakharova and Mishchenko, 2000). Such a case, however, would be striking anyway since it is reasonable to assume that it would not be possible to accurately mimic the measured infrared spectra of such needle-like or 


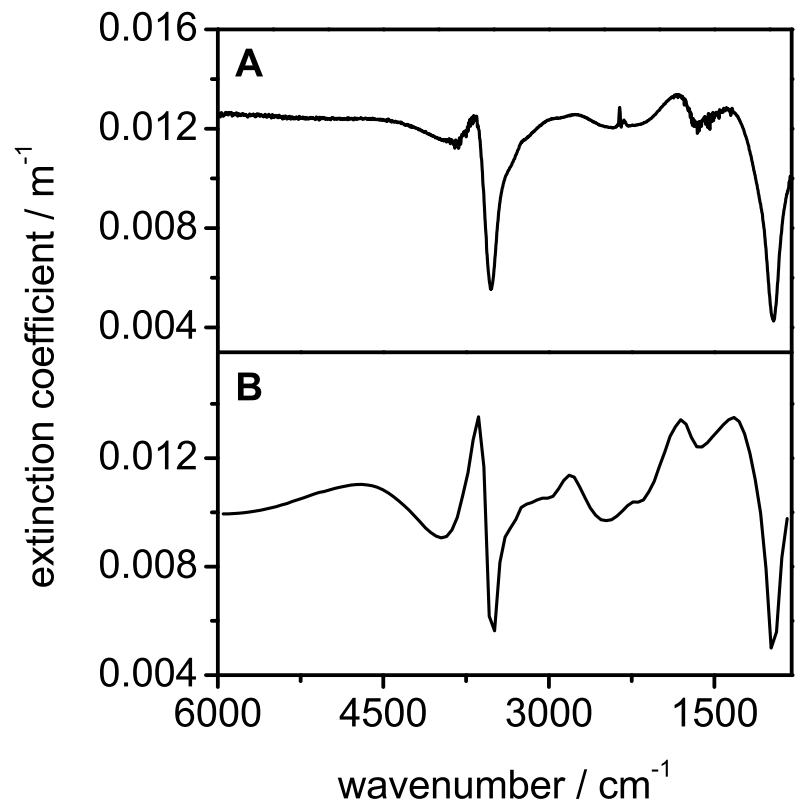

Fig. 7. Panel (A): Measured infrared extinction spectrum of ice crystals generated by deposition nucleation on mineral dust particles at $237 \mathrm{~K}$. The median size of the ice particles was approximately $13 \mu \mathrm{m}$ and their number concentration $30 \mathrm{~cm}^{-3}$. Panel (B): Calculated infrared extinction coefficients of log-normally distributed cylindrical ice particles of aspect ratio $\phi=1.0$ with the following size distribution parameters: $N=30 \mathrm{~cm}^{-3}, \sigma_{g}=1.15$, and $D=13 \mu \mathrm{m}$.

plate-like crystals assuming compact shape parameters like $\phi=1$ (Wagner et al., 2005b), in contrast to the fit results for moderately aspherical particles shown in Fig. 6. Anticipating the results of Sect. 4, we have never observed such behaviour in our AIDA expansion experiments.

For crystal sizes $>6 \mu \mathrm{m}$, one might suppose that the strong variation of the extinction cross sections with particle shape within the interference structure (Fig. 5, top panels) could offer a chance to infer information about the predominant ice crystal shape from the measured infrared spectra. But this is only possible on very rare occasions; see below. In most cases, the solution ambiguity of the retrieval makes the reliable determination of number concentration, size, and shape of the ice crystals extremely difficult (Kokhanovsky, 2005). Figure 7, panel (A), depicts a representative infrared spectrum for larger ice crystals, recorded during an AIDA expansion experiment at $237 \mathrm{~K}$ with mineral dust particles as ice nuclei. The spectrum shows the signature which we most frequently encountered during the expansion runs, being characterised by an almost constant extinction coefficient at nonabsorbing wavenumbers between 6000 and $4000 \mathrm{~cm}^{-1}$ and by the two distinct extinction minima (Christiansen bands) of large ice crystals at about 3500 and $950 \mathrm{~cm}^{-1}$ (Arnott et al., 1995; Schmitt and Arnott, 1999; Yang et al., 1997). Using the measured ice particle number concentration $\left(\sim 30 \mathrm{~cm}^{-3}\right.$, from WELAS) in combination with the simultaneously measured ice water content (see Sect. 2.2), we can estimate the diameter of the ice crystals to be about $13 \mu \mathrm{m}$, assuming a narrow, unimodal size distribution. But what can we independently learn from the analysis of the infrared spectrum? The only option which we can exclude is that the ice cloud is composed of a narrow distribution of compact crystals. In such a case, the oscillations in the plots of the extinction efficiency versus particle diameter would be transformed into the measured spectrum at wavenumbers $>3600 \mathrm{~cm}^{-1}$. Panel (B) of Fig. 7 exemplarily shows a calculated extinction spectrum for $\phi=1$ with $N=30 \mathrm{~cm}^{-3}, \sigma_{g}=1.15$, and $D=13 \mu \mathrm{m}$, clearly revealing the interference structure at non-absorbing wavenumbers. Under particular experimental conditions, as specified in the next section, we indeed observed such spectral signature in our measured spectra. This is a clear indication for a compact crystal shape and turns infrared extinction spectroscopy into a powerful tool for the precise determination of the size of the crystals, given that the frequency positions of the extinction maxima and minima are very sensitive to the particle size. In the majority of cases, however, the interference structure was strongly damped in our recorded spectra. Without any a priori information, one could think of at least three different scenarios which would provoke this spectral behaviour. Firstly, Fig. 5 has demonstrated that the oscillations in the extinction spectrum would vanish for an ice cloud composed of a broad variety of differently shaped crystals or of increasingly aspherical particles, even when assuming a narrow size distribution. Secondly, also for compact cylindrical shapes such a spectral signature could be reproduced, but only when assuming a sufficiently broad distribution of particle sizes, thereby also eliminating much of the interference structure. And thirdly, instead of a broad unimodal distribution, an appropriately chosen bi- or multimodal size distribution would result in the same effect, i.e., the flattening of the extinction maxima and minima. The diversity of possible solutions to the retrieval problem might confine the applicability of the FTIR measurements for the characterisation of ice clouds in such a case. This will be further elaborated and quantified in the next section by means of selected AIDA expansion runs, using the data of the optical particle counters as a benchmark.

\section{Results of AIDA ice nucleation experiments}

Following the outline of the preceding section, we will at first address the expansion experiments where small ice crystals with $D<6 \mu \mathrm{m}$ were generated. FTIR and WELAS data for two representative AIDA runs, one homogeneous freezing and one heterogeneous ice nucleation experiment, are shown in Fig. 8. They correspond to case studies B and C which were discussed in the context of the WELAS measurements in Sect. 2.2 (see Fig. 2). Series of FTIR extinction 
I
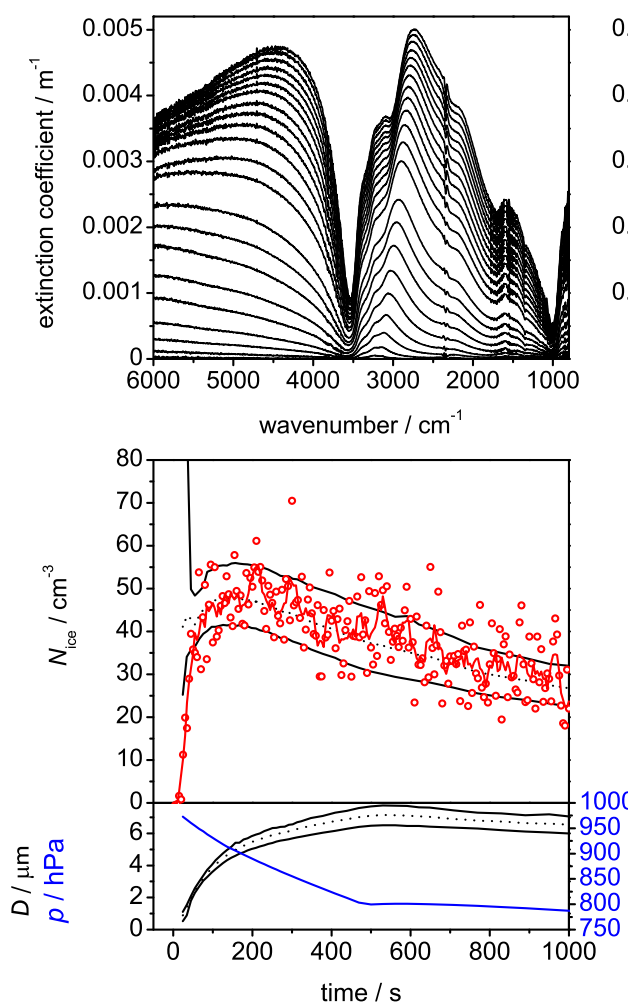

II
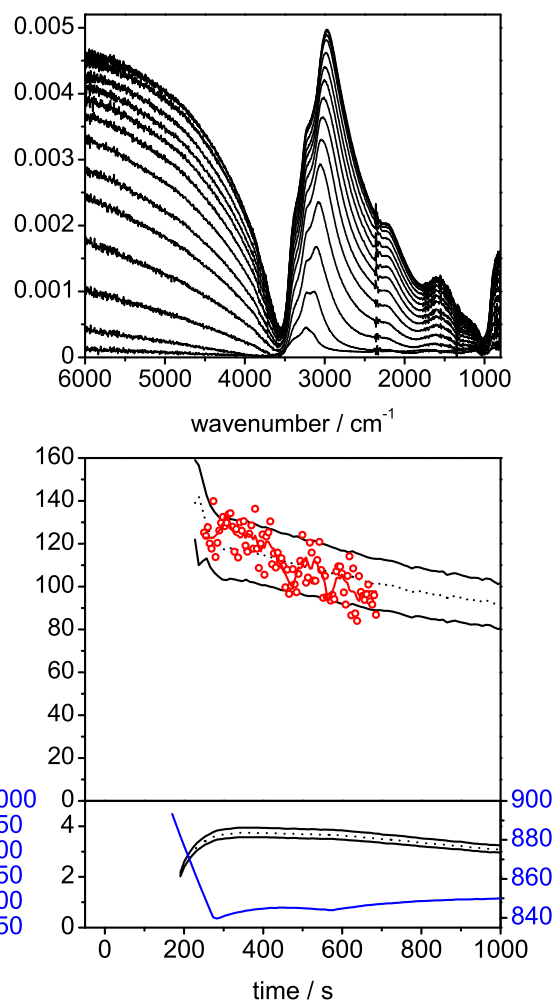

Fig. 8. FTIR and WELAS recordings for two AIDA expansion experiments: I (left column, corresponding to experiment B of Fig. 2): Heterogeneous ice nucleation on Saharan dust aerosol particles at 213 K. II (right column, corresponding to experiment C of Fig. 2): Homogeneous freezing of supercooled $\mathrm{H}_{2} \mathrm{SO}_{4} / \mathrm{H}_{2} \mathrm{O}$ solution droplets at $208 \mathrm{~K}$. The top panels show the series of infrared extinction spectra which were recorded during the expansion runs at time intervals of $10 \mathrm{~s}$. Comparisons between the time-dependent ice particle number concentrations which were deduced from the WELAS data and the FTIR extinction spectra are shown in the middle panels. Solid black lines indicate maximum and minimum $N_{\text {ice }}(t)$ from the FTIR retrieval; dotted black lines indicate $N_{\text {ice }}(t)$ averaged over all different shape distributions; see text for details. Red dots designate $N_{\text {ice }}(t)$ derived from the original $N_{\text {WELAS }}(t)$ records, as outlined in Sect. 2.2 (same data as in Fig. 2). The red lines are obtained by smoothing the $N_{\text {ice }}(t)$ WELAS data points (Savitsky-Golay filter). The bottom panels show the ice particle (equal-volume sphere) median diameters retrieved from the FTIR spectra vs. experimental time. Again, solid black lines are used for the maximum and minimum diameters and dotted black lines for the averaged values. No WELAS data are shown because aspherical particles cannot be properly sized by the instrument. Blue lines in the lowest panels represent AIDA pressure profiles during the expansion runs.

spectra which were recorded every $10 \mathrm{~s}$ during the ice nucleation events are shown in the top panels. They cover a time frame of $t=24-234 \mathrm{~s}$ for experiment I and $t=170-310 \mathrm{~s}$ for experiment II (from the bottom to the top spectra). The progressive change in the habitus of the extinction spectra, being more and more dominated by light scattering, nicely underlines the continuous growth of the ice crystals after the onset of ice nucleation. In the middle panels, we compare the WELAS ice particle measurements with the $N_{\text {ice }}(t)$ data which were retrieved from the infrared spectra. As described in Sect. 2.3, we have performed 126 individual Tmatrix fits for each spectrum, corresponding to different permutations of the mixing coefficients $a_{i}$ which define various shape distributions of cylindrical ice crystals. The fit results are displayed as follows: Solid black lines are envelopes of the highest and lowest ice particle number concentrations ob- tained from the 126 individual retrieval results, while the dotted black lines denote the averages for $N_{\text {ice }}(t)$ of all individual fit outcomes. As discussed in Sect. 3, $N_{\text {ice, max }}(t)$ usually corresponds to the fit with $a_{3}=1$ (i.e., $\phi=1$ ) whereas the lowest ice particle number concentrations were retrieved for pure $\phi=3$ crystals $\left(a_{5}=1\right)$. For all combinations of the mixing coefficients, a close agreement was found between measured and calculated infrared extinction spectra (see Figs. 6 and 9). Hence, the band width of the retrieval results, as spanned by $N_{\text {ice,max }}(t)$ and $N_{\text {ice,min }}(t)$, marks the extent of the solution ambiguity of the retrieval problem within the considered range of ice particle shapes (see Table 1 for comparison). For experiment I, $N_{\text {ice, max }}$ and $N_{\text {ice, min }}$ show a significant spread at the very beginning of the ice nucleation event. Here, the freshly nucleated ice crystals are still so small that their infrared extinction spectra are dominated by light absorption. 

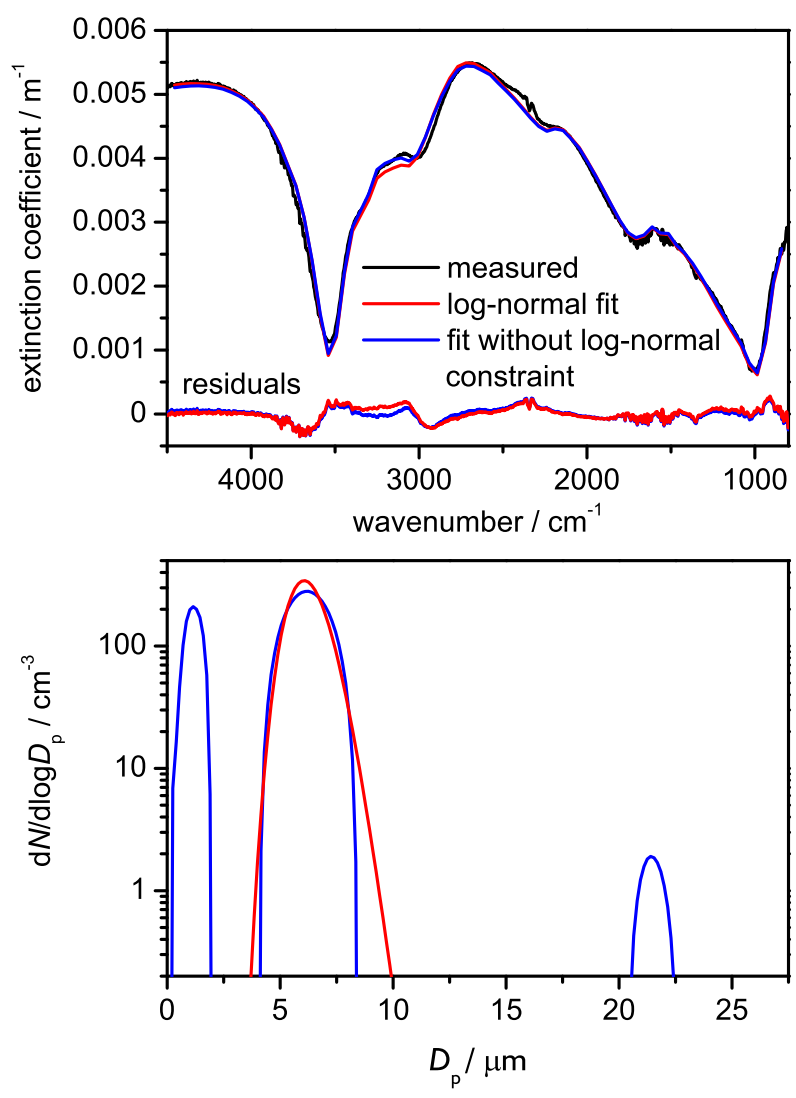

Fig. 9. Top panel: Measured infrared extinction spectra of heterogeneously nucleated ice crystals on Saharan dust particles at $213 \mathrm{~K}$; comparison with two best fit results for cylindrical ice crystals of aspect ratio $\phi=0.7$, (a) assuming a unimodal log-normal distribution function (red lines), and (b), relaxing the log-normal constraint in the size distribution retrieval (blue lines); see text for details. The spectral residuals are also shown. Bottom panel: Retrieved ice particle number size distributions for the scenarios (a) and (b).

As a result, the retrieval is sensitive to the volume concentration of ice but insensitive to the individual parameters of the log-normal size distribution, regardless of the assumed ice crystal shape. Even for a fixed aspect ratio, similar optical depths can be reproduced by different combinations of $N, \sigma_{g}$, and $D$ (Echle et al., 1998), showing that the fit is arbitrary for spectra with a weak scattering contribution. Unique fit results in terms of the log-normal parameters were only obtained for ice crystal sizes larger than $1.5 \mu \mathrm{m}$. For experiment II, we have therefore omitted the initial time period of the ice nucleation event with $D<1.5 \mu \mathrm{m}$ in the plots of the FTIR retrieval results. For both expansion runs shown in Fig. 8, the ice crystal number concentrations deduced from the WELAS records nicely agree with the infrared retrievals. Further inter-comparisons between FTIR retrievals and ice particle number concentrations measured with our OPCs in the $D<6 \mu \mathrm{m}$ size range are shown in Möhler et al. (2006),
Figs. 5 and 6, confirming the good agreement between the $N_{\text {ice }}(t)$ data derived from both measurement techniques. For the vast majority of AIDA expansion experiments with single ice nucleation events (irrespective of the actual temperature, cooling rate, or nature of the seed aerosol), leading to ice crystal median sizes of at most 6-7 $\mu \mathrm{m}$, the $N_{\text {ice }}(t)$ time profiles obtained from the FTIR retrieval and the OPCs were found to be in good agreement, provided that $N_{\text {ice }}$ could be reliably inferred from the PCS or WELAS data sets as described in Sect. 2.2. An example for a slight mismatch between the FTIR and WELAS measurements, most probably due to instrumental problems of the OPC, will be shown below.

In summary, our results indicate that the approach of modelling aspherical ice crystals by circular cylinders with moderate aspect ratios of $\phi=0.5-3.0$ is appropriate for retrieving number densities and diameters from the FTIR extinction spectra of smaller ice crystals over a wide range of experimental conditions. It is well known that the ice crystal growth, and thereby the ice crystal habit, strongly varies with temperature and ice supersaturation (e.g. Bailey and Hallett, 2004; Libbrecht and Yu, 2001). To deduce the ice crystal habit diagrams from laboratory measurements, the ice crystals are usually grown at nearly constant temperature and humidity conditions. In contrast, the ice crystals which are formed during an AIDA expansion experiment experience variable growth conditions due to the changing water vapour concentration and temperature in the course of the expansion. Nevertheless, we can state that within the broad range of different ice cloud formation conditions which were covered by our experiments (temperature range from 238 to $195 \mathrm{~K}$, critical ice supersaturations ranging from a few percent for very ice-active mineral dust samples like Arizona test dust up to $70 \%$ for homogeneously nucleating sulphuric acid droplets at $195 \mathrm{~K}$ ) rather compact ice crystal habits were predominantly formed within growth times of the order of a few minutes. But due to the solution ambiguity of the FTIR retrieval, we are unable to further distinguish between the formation of e.g. thick plates $(\phi=2-3)$ or short columns $(\phi=0.5)$. Further information about the actual shape of small-sized ice crystals may be obtained in future chamber studies employing the second generation probe SID2 (http://strc.herts.ac.uk/pi/over.html). This new device should be capable of classifying the shape of cloud particles down to micrometer sizes.

But note that the analysis of our infrared spectra was constrained by the independent information that only single ice nucleation events had occurred, yielding unimodal size distributions of ice crystals which could be approximated by log-normal distribution functions. We have also tested the effect of relaxing the single mode constraint on the retrieval result. Figure 9 (top panel) compares a measured infrared extinction spectra, selected from the spectra series of experiment $\mathrm{I}(t=314 \mathrm{~s})$, with the best log-normal fit result for $\phi=0.7$ (i.e., $a_{2}=1$ ). The retrieved ice crystal size distribution 
is shown in red in the bottom panel. An ice particle number concentration of $48 \mathrm{~cm}^{-3}$ and an ice volume concentration of $5980 \mu \mathrm{m}^{3} / \mathrm{cm}^{3}$ were retrieved. The spectral residuals shown in the upper panel are typical for all our fit results. They are attributed to the limited precision of the employed refractive index data sets and the inevitable approximation of the actual ice crystal shapes in the retrieval. Also shown in Fig. 9 is the fit result for $\phi=0.7$ when relaxing the log-normal constraint on the size distribution, using the iterative algorithm from Arnott et al. (1997b) with a smoothing factor of 0.3. By this approach, the small spectral mismatch which was apparent for the log-normal fit is further reduced, especially in the regime of the $\mathrm{O}-\mathrm{H}$ stretching mode at around $3250 \mathrm{~cm}^{-1}$. In return, pseudo modes of smaller and larger ice crystals appear in the retrieved size distribution, similar to the results from Liu et al. (1999). The deduced ice volume concentration is only weakly affected by these additional ice crystal modes $\left(V_{\text {ice }}=6200 \mu \mathrm{m}^{3} / \mathrm{cm}^{3}\right)$, the retrieved overall ice particle number concentration, however, is severely distorted by the additional small particle mode $\left(N_{\text {ice }}=129 \mathrm{~cm}^{-3}\right)$. Thus, a retrieval algorithm which allows multi-modal particle size distributions should not be employed as the standard inversion technique unless there is independent evidence that more than one mode is involved. Rather, the additional degrees of freedom in this approach may give rise to an unphysical compensation for inaccurate refractive index data sets and/or particle shape representations.

The good agreement between the WELAS data and the FTIR retrieval results with respect to $N_{\text {ice }}$ provides a reliable basis for the ongoing ice nucleation experiments in the AIDA chamber. Having demonstrated the robustness of the FTIR retrieval procedure by the comparison with the WELAS data, one can rely on the FTIR data whenever it is impossible to accurately deduce $N_{\text {ice }}$ from the WELAS measurements, e.g. when the interference by un-activated seed aerosol particles is significant. For example, the ice particle number concentrations for experiment II (right panel in Fig. 8) cannot be inferred from WELAS for the complete activation period but only for the time interval $220<t / \mathrm{s}<650 \mathrm{~s}$. Another typical scenario where the WELAS data might be in error is displayed in Fig. 10, showing the time-dependent ice particle number concentrations for a heterogeneous ice nucleation experiment with Saharan dust particles as seed aerosol at $T=197 \mathrm{~K}$. Due to the low temperature, the ice crystals only grew to median sizes of about $2 \mu \mathrm{m}$ in the course of the expansion. In this example, $N_{\text {ice }}(t)$ from WELAS falls below the band width of the FTIR retrieval results, even when considering the following adjustments: (i) As outlined in Sect. 2.3, all T-matrix calculations were performed using infrared complex refractive indices for ice at $210 \mathrm{~K}$. Therefore, the temperature dependence of the optical constants might infect the retrieval results for the $197 \mathrm{~K}$ expansion run. As a check, we have calculated the $M \times N$ matrix of extinction cross sections for one selected aspect ratio $(\phi=0.7)$ also with the Clapp et al. (1995) refractive index

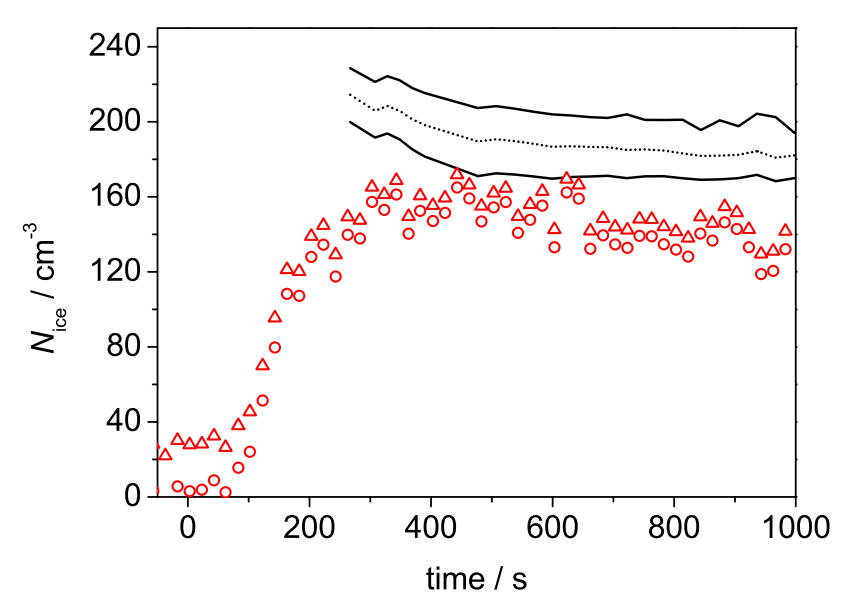

Fig. 10. Heterogeneous ice nucleation on Saharan dust particles at 197 K. Comparison between the time-dependent ice particle number concentrations which were deduced from the WELAS recordings and the FTIR extinction spectra. Similar to Fig. 8, solid black lines indicate $N_{\text {ice,max }}(t)$ and $N_{\text {ice,min }}(t)$ from the FTIR retrieval, the dotted black line displays $N_{\text {ice }}(t)$, averaged over all considered shape distributions; see text for details. Red triangles designate the total particle number concentration, $N_{\text {WELAS }}(t)$, measured with WELAS in the course of the expansion run. $N_{\text {ice }}(t)$, as derived from $N_{\text {WELAS }}(t)$ using Eq. (2), is marked by red circles.

data set for $190 \mathrm{~K}$. A comparison between the fit results for $\phi=0.7(210 \mathrm{~K})$ and $\phi=0.7(190 \mathrm{~K})$ revealed that the retrieved ice particle number concentrations for the $190 \mathrm{~K}$ data set are typically about $5 \%$ lower than for the $210 \mathrm{~K}$ data set. This reflects that the intensity of the $\mathrm{O}-\mathrm{H}$ stretching mode in the spectrum of the imaginary index $k$ is increasing with decreasing temperature. A constant factor of 0.95 was therefore used to scale all FTIR retrieval results for $N_{\text {ice }}(t)$ which are shown in Fig. 10. Although the onset of ice nucleation already occurred at $t=100 \mathrm{~s}$, the FTIR fit results are only plotted for $t>250 \mathrm{~s}$ where the ice crystals have grown to median sizes greater than $1.5 \mu \mathrm{m}$, thus allowing for an unambiguous retrieval of the size distribution parameters. (ii) In addition to the ice particle number concentrations (circles) which were deduced from the WELAS records using Eq. (2), also the total WELAS number concentrations $N_{\text {WELAS }}(t)$ (triangles) are presented in Fig. 10. The $N_{\text {WELAS }}(t)$ values define the upper limit of $N_{\text {ice }}(t)$ when all dust particles which are visible to WELAS at $t=0$ would act as ice nuclei during the expansion. But even then, as evident from Fig. 10, the WELAS records would slightly underestimate the ice particle number concentrations retrieved from the FTIR spectra. A possible explanation would be that highly aspherical ice crystals with aspect ratios exceeding the regime covered by the FTIR retrieval procedure have been formed. But this contrasts with the fact that all infrared spectra of this expansion run could be accurately fitted employing moderate aspect ratios. It is more likely that, due to the small sizes of the nucleated ice crys- 

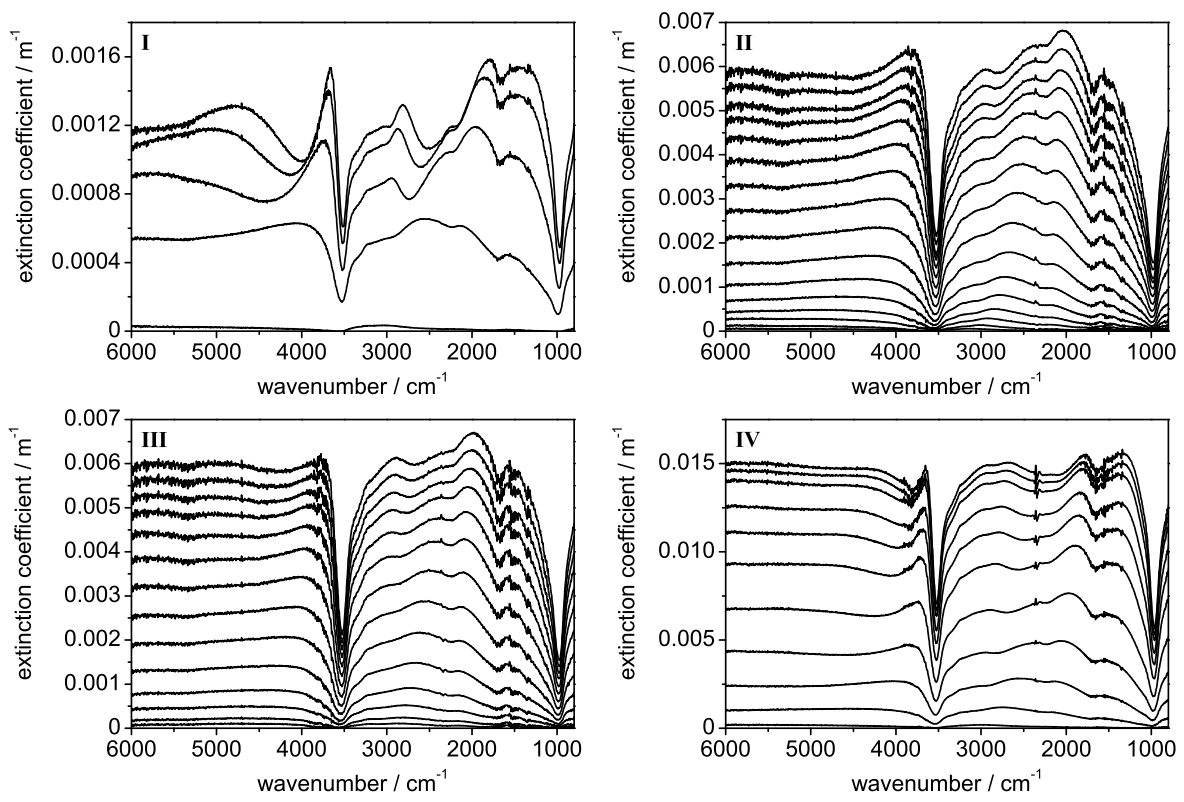

Fig. 11. Series of infrared extinction spectra from the initial period of four different AIDA expansion runs, recorded at time intervals of $40 \mathrm{~s}$ for experiment I, and of $10 \mathrm{~s}$ for experiments II, III, and IV. Exp. I: Homogeneous freezing of supercooled $\mathrm{H}_{2} \mathrm{SO}_{4} / \mathrm{H}_{2} \mathrm{O}$ solution droplets at 211 K. Exp. II: Heterogeneous ice nucleation on Asian dust particles at 222 K. Exp. III: Heterogeneous ice nucleation on Saharan dust particles at $223 \mathrm{~K}$. Exp. IV: Heterogeneous ice nucleation on Arizona test dust particles at $237 \mathrm{~K}$.

tals, a significant fraction of the ice cloud could not be detected by WELAS because the "apparent" crystal sizes were smaller than $1 \mu \mathrm{m}$, thereby leading to an underestimation of the actual ice particle number concentration.

Figure 11 shows series of FTIR spectra recorded during four different AIDA expansion runs which resulted in the formation of larger ice crystals with $D>6 \mu \mathrm{m}$. With respect to the spectral habitus of the extinction spectra, experiment $\mathrm{I}$, a homogeneous freezing event of supercooled $\mathrm{H}_{2} \mathrm{SO}_{4} / \mathrm{H}_{2} \mathrm{O}$ solution droplets at $T=211 \mathrm{~K}$, is clearly different. Here, in contrast to all other spectra recordings, a characteristic interference structure at non-absorbing wavenumbers $>3600 \mathrm{~cm}^{-1}$ is developing in the course of the expansion. As pointed out in Sect. 3, this is a rare occasion where FTIR extinction spectroscopy allows for an unambiguous determination of size and shape of the nucleated ice crystals. This is demonstrated in Fig. 12a, where the uppermost infrared spectrum from this series is compared to the best $\mathbf{T}$-matrix fit results, based on different assumptions about the particle shape. For an aspect ratio of $\phi=1$, measured and calculated infrared spectra are in excellent agreement. The retrieved parameters for the log-normal size distribution are $N=3.7 \mathrm{~cm}^{-3}, \sigma_{g}=1.12$, and $D=12.7 \mu \mathrm{m}$. Thus, only for a narrow number size distribution of compact crystals the oscillations in the extinction spectrum are accurately mimicked. The quality of the Mie fit as well as the T-matrix fit results for $\phi=0.5$ and $\phi=2$ are distinctly poorer in comparison with the $\phi=1.0$ cylindrical shape, underlining the peculiarity of this retrieval problem where only a single size-shape representation is adequate for reproducing the measured infrared spectrum. The formation of such uniform ice clouds, being predominantly composed of very compact ice crystals, was rarely observed in the AIDA ice nucleation experiments. Interestingly, most of the FTIR spectra featuring the characteristic interference structure were recorded in a series of experiments on the homogeneous freezing of $\mathrm{H}_{2} \mathrm{SO}_{4} / \mathrm{H}_{2} \mathrm{O}$ solution droplets at temperatures between 220 and $195 \mathrm{~K}$ which were conducted at reduced chamber pressure, typically by pumping from 180 to $110 \mathrm{hPa}$. Most other expansion runs, which were started at $1000 \mathrm{hPa}$, did not show the characteristic interference structure. This implies that the shapes of the nucleated ice crystals are strongly dependent on pressure and/or temperature.

The good match of the $\phi=1$ spectrum must indeed be emphasised, since it also points to the accuracy of the employed infrared refractive indices over the complete wavenumber regime, keeping in mind that the pronounced interference structure provides little scope to correct for potential mistakes in the optical constants by transforming them into the retrieved particle size distribution. This can be seen in the fifth and sixth panel of Fig. 12a, where we have repeated the fit for $\phi=1$ with different refractive index data sets as input for the T-matrix calculations. In panel five, we have used the original Clapp et al. (1995) refractive indices instead of replacing them in the $1500-800 \mathrm{~cm}^{-1}$ regime with the recent Zasetsky et al. (2005) data. For the fit shown in panel six, we have employed the popular compilation of optical constants for ice by Warren (1984). Note that this compilation includes only a single measurement at $T=266 \mathrm{~K}$ for the mid-infrared 
regime. Both modified refractive index data sets lead to a poorer agreement between measured and calculated spectra compared to the fit result shown in the top panel, particularly in the atmospheric window region from $1500-800 \mathrm{~cm}^{-1}$. This regime is shown in expanded view in Fig. 12b, covering the Christiansen band of ice at around $970 \mathrm{~cm}^{-1}$, i.e., a minimum in the extinction coefficients which can be ascribed to reduced scattering cross sections because the real part of the complex refractive index approaches unity (Arnott et al., 1995). When using the Clapp et al. (1995) data set, the magnitude of the Christiansen effect at $970 \mathrm{~cm}^{-1}$ is underestimated by almost $30 \%$ compared to the measured spectrum. When employing the Warren (1984) compilation, even the spectral habitus of the Christiansen band is not accurately reproduced. The minimum in the extinction coefficients is shifted by $>30 \mathrm{~cm}^{-1}$ to lower wavenumbers in comparison with the measurement. As stated in Sect. 2.3, the poor match with the Clapp et al. (1995) data at wavenumbers below $1500 \mathrm{~cm}^{-1}$ can be traced back to a procedural inaccuracy when deriving the refractive index data sets via the KramersKronig integration. The poor quality of the Warren (1984) fit just reflects the pronounced temperature dependence of the optical constants of ice. In view of the tremendous efforts which are made for calculating shape-dependent extinction properties of ice crystals (Baran, 2005; Kokhanovsky, 2004; Yang et al., 2005), the results from Fig. 12b make clear that the same attention should also be paid to the appropriate choice of the employed infrared refractive indices.

For experiments II, III, and IV (Fig. 11), the interference structure in the FTIR spectra is much less pronounced. As already suggested in Sect. 3, many different size-shape distributions of ice crystals are able to reproduce this spectral signature, which gives rise to ambiguous retrieval results. This will be demonstrated in detail by means of experiment II (heterogeneous ice nucleation on Asian dust particles at $222 \mathrm{~K}$ ). Panel (A) of Fig. 13 shows our typical intercomparison between the ice crystal number concentrations which were deduced from the WELAS and FTIR measurements. Note that the spreading of the FTIR retrieval results is much larger compared to the previous fit results for expansion runs where smaller ice crystals were formed. In the present example, $N_{\text {ice, max }}(t)$ is almost twice as high as $N_{\text {ice,min }}(t)$. The highest ice particle number concentrations were retrieved for the most compact crystal shape, i.e., $\phi=1$. Panel (B) compares an infrared extinction spectrum from this expansion run (recorded at $t=173 \mathrm{~s}$ ) with the best fit result for $\phi=1$. The retrieval yields $N=41.4 \mathrm{~cm}^{-3}, \sigma_{g}=1.40$, and $D=6.7 \mu \mathrm{m}$. The lowest ice particle number concentrations were typically retrieved for all those combinations of the mixing coefficients $a_{i}$ where the oscillations in the plots of the extinction efficiencies versus ice crystal size for wavenumbers $>3600 \mathrm{~cm}^{-1}$ are largely smoothed. This is exemplified in panel (D) for a shape distribution with $a_{1}=0$, $a_{2}=0, a_{3}=0.2, a_{4}=0.2$, and $a_{5}=0.6$. The corresponding fit to the measured spectrum is displayed in panel (C). Here,
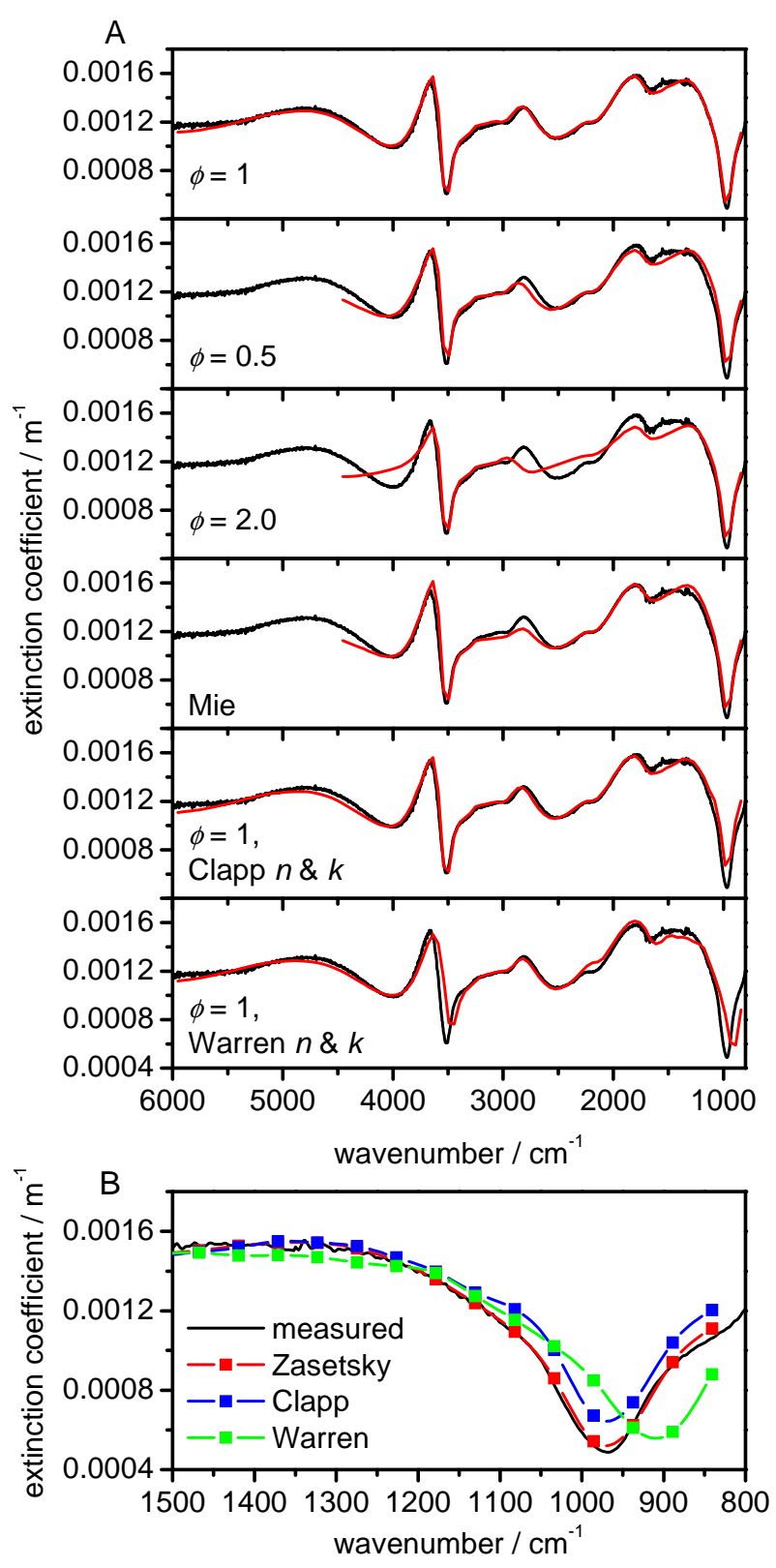

Fig. 12. Part A: Measured infrared extinction spectrum of ice crystals (black lines), generated via homogeneous freezing of supercooled sulphuric acid solution droplets at $211 \mathrm{~K}$; comparison with best fit results for different assumptions on the particle shape and different infrared refractive indices (red lines); see text for details. Part B: Expanded view of the 1500-800 wavenumber regime for the fits shown in panels 1,5 , and 6 of part A.

$N=24.0 \mathrm{~cm}^{-3}, \sigma_{g}=1.08$, and $D=9.2 \mu \mathrm{m}$ were retrieved. Both T-matrix fits are in fair agreement with the measured spectrum (with nearly identical root-mean squares deviations); although the match is clearly not as perfect as for the spectra of smaller ice crystals; see Fig. 9. Hence, our measured spectrum can either be reproduced by (i) a narrow distribu- 

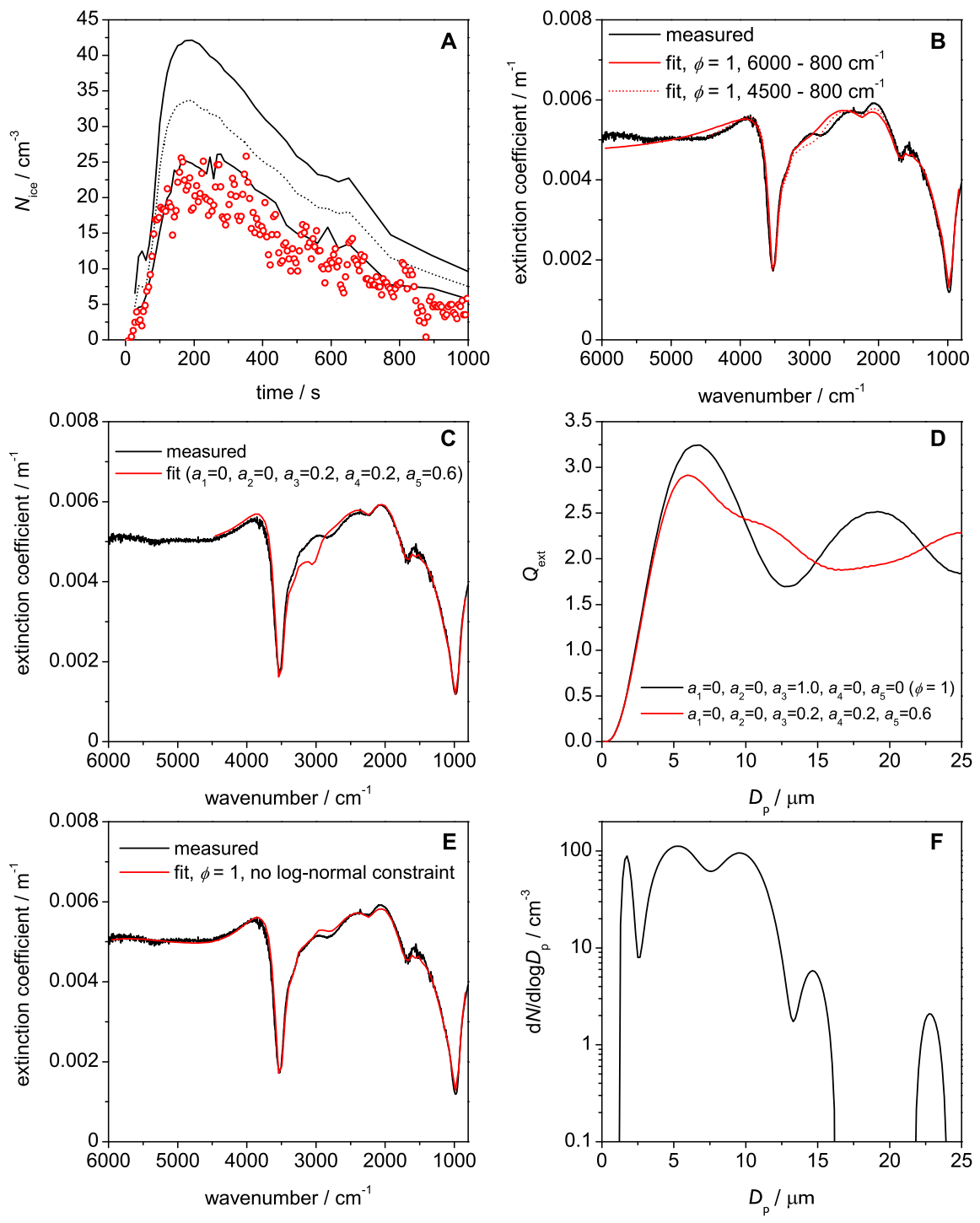

Fig. 13. Heterogeneous ice nucleation on Asian dust aerosol particles at $222 \mathrm{~K}$. Panel (A): Comparison between the time-dependent ice particle number concentrations which were deduced from the WELAS data and the FTIR extinction spectra. Solid black lines indicate maximum and minimum $N_{\text {ice }}(t)$ from the FTIR retrieval, the dotted black line denotes $N_{\text {ice }}(t)$ averaged over all different shape distributions. Red dots designate $N_{\text {ice }}(t)$ derived from the original $N_{\text {WELAS }}(t)$ records. Panel (B): Measured infrared extinction spectra at $t=173 \mathrm{~s}$ in comparison with best fit results for $\phi=1$. Panel (C): Same as in panel (B), but using a shape distribution of cylindrical ice crystals to fit the measured spectrum. Panel (D): Plots of the extinction efficiencies at $4456.8 \mathrm{~cm}^{-1}$ versus ice crystal equal-volume sphere diameter for the two different shape distributions which are employed for the fits in panels (B) and (C). Panel (E): Same as in panel (B), but relaxing the log-normal constraint in the size distribution retrieval. Panel (F): Retrieved ice crystal size distribution for the fit in panel (E).

tion of larger ice crystals when assuming a shape distribution with sufficiently damped oscillations in the $Q_{\text {ext }}$ curve (so to say an a priori flattening of the interference structure), or by (ii) a broad distribution of ice crystals when assuming compact crystal shapes. Note that in this case the retrieved median diameter of the particle size distribution, accompanied by an increase in the number concentration, shifts to much smaller sizes in order to preserve the ice volume concentration. Also shown in panel (B) is the best fit result for $\phi=1$ when including the complete wavenumber range from $6000-800 \mathrm{~cm}^{-1}$ in the fit. This puts even more weight on the accurate reproduction of the flattened extinction profile at non-absorbing wavenumbers, implicating that an even broader distribution of ice crystals is retrieved $\left(N=47.0 \mathrm{~cm}^{-3}, \sigma_{g}=1.49\right.$, and $\left.D=6.1 \mu \mathrm{m}\right)$. Only by comparing with the WELAS records, which closely follow the $N_{\text {ice, } \min }(t)$ retrieval results, can we conclude that the assumption of compact crystal shapes would lead to a sub- 

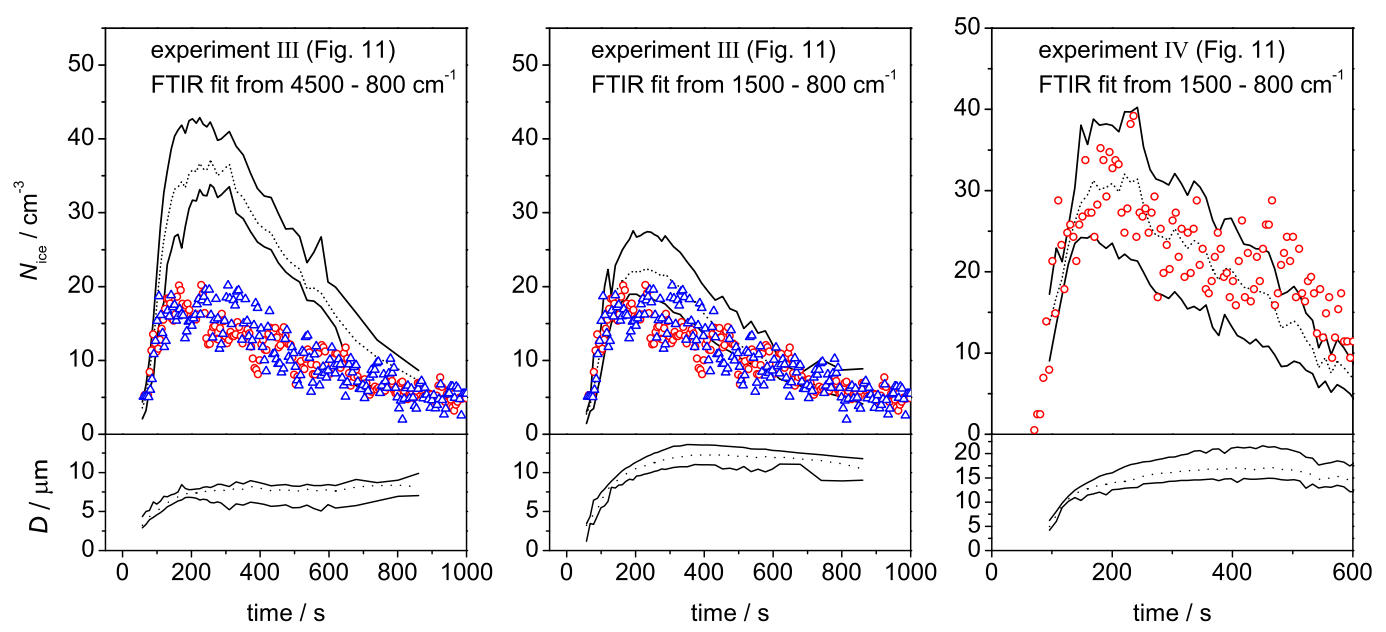

Fig. 14. Upper panels: Comparisons between ice particle number concentrations which were deduced from the OPCs and the FTIR extinction spectra in the course of two different AIDA expansion experiments. Left panel: Heterogeneous ice nucleation on Saharan dust particles at $223 \mathrm{~K}$ (exp. III in Fig. 11). Here, the FTIR retrieval was applied to the complete wavenumber range from $4500-800 \mathrm{~cm}^{-1}$. Middle panel: Same experiment as in the left panel, but confining the FTIR retrieval to the $1500-800 \mathrm{~cm}^{-1}$ regime. Right panel: Heterogeneous ice nucleation on Arizona test dust particles at $237 \mathrm{~K}$ (exp. IV in Fig. 11, FTIR fit from $1500-800 \mathrm{~cm}^{-1}$ ). In each panel, solid black lines indicate maximum and minimum $N_{\text {ice }}(t)$ from the FTIR retrieval, the dotted black lines denote $N_{\text {ice }}(t)$ averaged over all different shape distributions. Red dots designate $N_{\text {ice }}(t)$ derived from the original $N_{\text {WELAS }}(t)$ records. For experiment III, SID data were also available (blue triangles). Panels in the bottom row show ice particle (equal-volume sphere) median diameters retrieved from the FTIR spectra. Again, solid black lines are used for the maximum and minimum diameters, and dotted black lines for the averages.

stantial overestimation of the actual ice particle number concentration in this particular expansion run. In addition, the slight spectral mismatch between measured and calculated extinction spectra might prompt to relax the log-normal constraint in the size distribution retrieval. The best fit result for $\phi=1$ when employing the iterative algorithm from Arnott et al. (1997b) is shown in panel (E), the corresponding ice particle size distribution is displayed in panel (F). Obviously, our measured spectrum can be perfectly reproduced with an appropriately chosen multimodal size distribution. However, it is rather unlikely that such a complex size distribution was formed. Furthermore, the multimodal fit yields an ice particle number concentration of $62 \mathrm{~cm}^{-3}$, almost three times higher than the reference value from the OPC. Only when retrieving size distributions of strictly spherical particles, an improvement of the fit result by a multimodal fit might be meaningful (Walters, 1980). For aspherical particles, in particular for an ice cloud composed of various, partially irregular shapes, slight discrepancies between measured and calculated infrared spectra are virtually inevitable. This is especially true for non-absorbing spectral regions and ice crystal sizes larger than the first interference maximum, where the extinction cross sections are dramatically varying with the particle shape (see Fig. 5).

The preceding discussion showed that retrievals of ice particle number densities from FTIR spectra become more unreliable for larger ice crystals compared to particle diameters less than $6 \mu \mathrm{m}$. Figure 14, left panel, shows another inter- comparison between $N_{\text {ice }}(t)$ measured by the OPCs (WELAS and SID data are displayed) and the FTIR retrievals, now for experiment III (heterogeneous ice nucleation on Saharan dust particles at $223 \mathrm{~K}$ ). Here, the FTIR retrieval results for all considered shape distributions are significantly higher than the reference measurements; precisely, $N_{\text {ice }}(t)$ is overestimated by factors of two to three. Even for shape distributions with damped oscillations in the $Q_{\text {ext }}$ curve, which again usually gave rise to the $N_{\text {ice,min }}(t)$ retrieval results, rather broad distributions of ice crystals with $\sigma_{g}>1.6$ were deduced, thereby also distorting the retrieval results for the median sizes and number concentrations of the ice crystals (e.g., at $t=351 \mathrm{~s}$, we retrieved $N=30.8 \mathrm{~cm}^{-3}, \sigma_{g}=1.71$, and $D=8.1 \mu \mathrm{m}$ for $a_{1}=0.2, a_{2}=0.2, a_{3}=0.2, a_{4}=0.2$, and $\left.a_{5}=0.2\right)$. To match $N_{\text {ice }}(t=351 \mathrm{~s})$ measured by WELAS and SID (about $15 \mathrm{~cm}^{-3}$ ), the actual size distribution should feature a much smaller mode width in combination with a significantly larger median ice crystal size. An explanation for this retrieval error might be that the interference structure in the recorded infrared spectra at wavenumbers $>3600 \mathrm{~cm}^{-1}$ is even more flattened in this expansion run compared to experiment II. Thus, even the small oscillations which are still visible in the $Q_{\text {ext }}$ curve for shape distributions like $a_{1}=0.2$, $a_{2}=0.2, a_{3}=0.2, a_{4}=0.2$, and $a_{5}=0.2$ or $a_{1}=0, a_{2}=0, a_{3}=0.2$, $a_{4}=0.2$, and $a_{5}=0.6$ have to be levelled out in the course of the retrieval by increasing the mode width of the size distribution in order to accurately mimic the flat extinction profile of the measured infrared spectra at non-absorbing wavenum- 

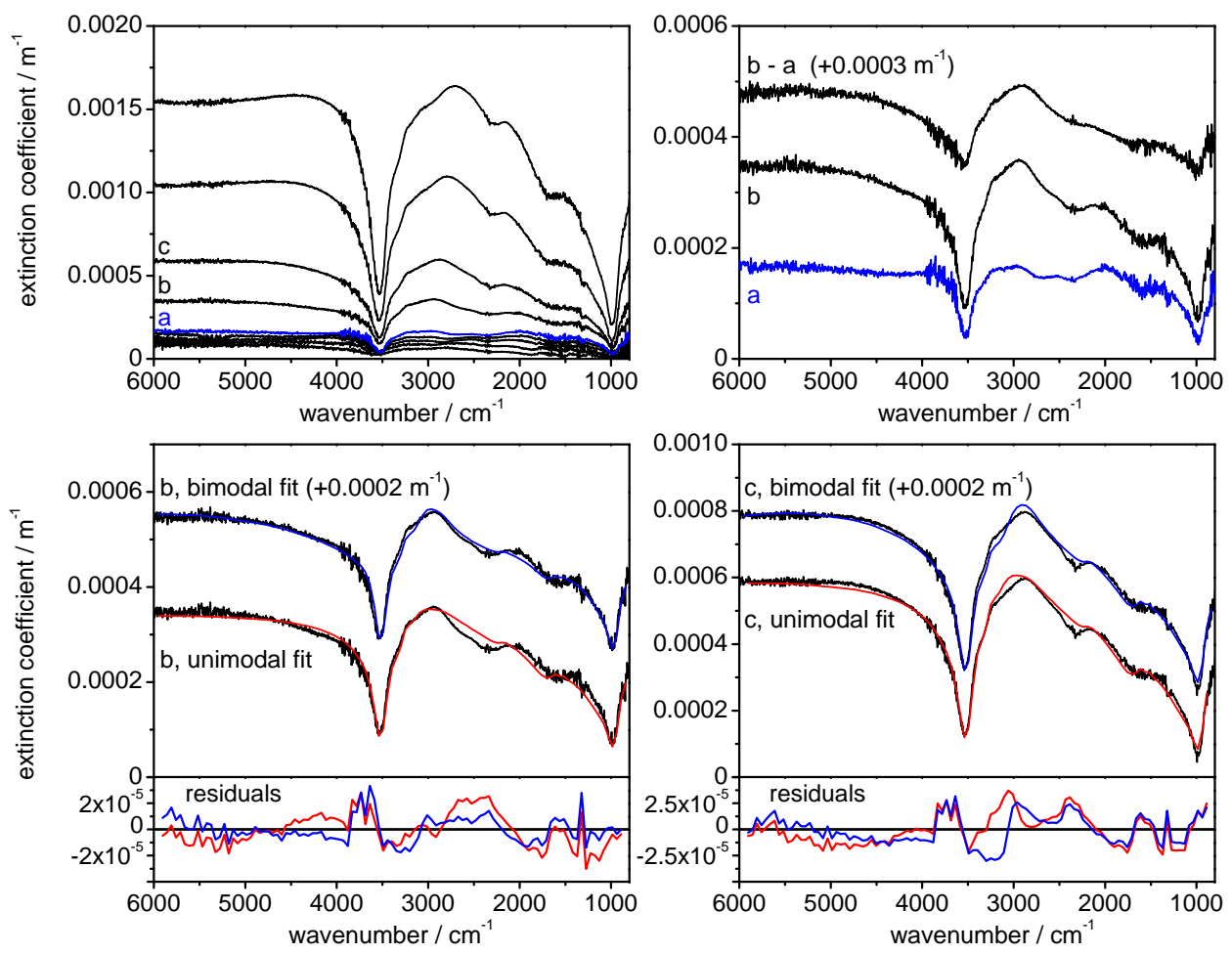

Fig. 15. Top left panel: Series of FTIR extinction spectra, recorded at time intervals of $10 \mathrm{~s}$ during an AIDA expansion run at $T=225 \mathrm{~K}$, using an external mixture of Saharan dust particles and supercooled $\mathrm{H}_{2} \mathrm{SO}_{4} / \mathrm{H}_{2} \mathrm{O}$ solution droplets as seed aerosol. Spectra a and b from this series, as well as their difference spectrum, are shown on an expanded scale in the top right panel. Bottom left and right panels: Measurements $b$ and $\mathrm{c}$ from the spectra series shown on top (black lines) in comparison with the best T-matrix fit results for unimodal (red lines) and bimodal (blue lines) log-normal size distributions of ice crystals; see text for details. The spectral residuals of the uni- and bimodal fits are attached below.

bers. In order to obtain an accurate retrieval result for $N_{\text {ice }}$ in such a case, one would have to employ a shape distribution of ice crystals for which the oscillations in the $Q_{\text {ext }}$ curves were even more dampened, e.g. by considering even more aspherical particle shapes as in the present study. A further solution to this retrieval problem might be the following. In principle, the retrieval approach benefits from the fact that our extinction measurements cover a broad infrared frequency regime. For certain scenarios (e.g. experiment I in Fig. 11), very reliable retrieval results can be obtained by including the complete experimentally accessible wavenumber range in the T-matrix fits, particularly also the regime of non-absorbing wavenumbers $>3600 \mathrm{~cm}^{-3}$. But if the generated ice clouds do not feature a uniform, pristine ice crystal shape, but instead include a diversity of pristine and/or complex habits, it becomes impossible to find an accurate model which reproduces the extinction spectra of larger crystals at wavenumbers $>3600 \mathrm{~cm}^{-3}$, in view of the dramatic variation of $C_{\text {ext }}$ with particle shape (see top left panel of Fig. 5). However, even for ice crystal sizes of 10-15 $\mu \mathrm{m}$, the first interference maximum is not reached when introducing an upper wavenumber limit of $\tilde{v}=1500 \mathrm{~cm}^{-1}$. The bottom left panel of Fig. 5 clearly reveals that the depen- dence of $C_{\text {ext }}$ on the particle shape is significantly reduced for $\tilde{v}<1500 \mathrm{~cm}^{-1}$. We have therefore repeated the $\mathbf{T}$-matrix fits for experiment III (middle panel of Fig. 14), this time confining the wavenumber range of the retrievals to 1500 $800 \mathrm{~cm}^{-1}$. The same method was used to deduce $N_{\text {ice }}(t)$ from the FTIR spectra series of experiment IV (heterogeneous ice nucleation on Arizona test dust particles at $237 \mathrm{~K}$, right panel of Fig. 14). For experiment III, the modified approach leads to a much better agreement of the FTIR retrievals with the OPC data. The derived $N_{\text {ice, } \min }(t)$ curve closely matches the WELAS data points, and also the averaged $N_{\text {ice }}(t)$ data from the FTIR retrieval are still in good accordance with the reference values. Only for very compact shapes, representative of the $N_{\text {ice, max }}(t)$ retrieval results, the actual ice particle number concentrations are noticeably overestimated. From the infrared spectrum recorded at $t=351 \mathrm{~s}$, we now retrieved the size distribution parameters $N=18.4 \mathrm{~cm}^{-3}, \sigma_{g}=1.16$, and $D=12.1 \mu \mathrm{m}$ for $a_{1}=0.2, a_{2}=0.2$, $a_{3}=0.2, a_{4}=0.2$, and $a_{5}=0.2$. As suggested above, the actual size distribution of the ice crystals indeed features a narrow mode of larger particles. For experiment IV, the WELAS records are nicely embedded within the band width of the FTIR retrieval results spanned by $N_{\text {ice, max }}(t)$ and $N_{\text {ice, min }}(t)$. 
Hence, the same reasonable agreement between the OPCs and the FTIR retrievals for $N_{\text {ice }}(t)$, as observed for ice crystals with $D<6 \mu \mathrm{m}$, can also be obtained for larger ice crystal sizes, but only when limiting the wavenumber range of the $\mathbf{T}$ matrix fits to a regime where the dependence of the extinction cross sections on the particle shape is less pronounced.

Remembering that a unimodal distribution of ice crystals might be falsely classified as a bi- or multimodal size distribution in the FTIR retrieval, we want to consider as a final example an AIDA expansion experiment with a dual nucleation event, which really resulted in the formation of a bimodal number size distribution of ice particles. Figure 15, top left panel, displays a series of FTIR extinction spectra recorded during an expansion run at $225 \mathrm{~K}$, using as seed aerosol an external mixture of $\sim 35 \mathrm{~cm}^{-3}$ Saharan dust particles and $400 \mathrm{~cm}^{-3}$ supercooled sulphuric acid solution droplets. Because of the large difference in nucleation thresholds (critical ice saturation ratios of about 1.2 and 1.5 , respectively), two ice nucleation events occurred in succession. In the first event ice nucleated heterogeneously on the dust particles, while in the second event a significant fraction of the sulphuric acid droplet aerosol froze homogeneously. Spectra a and $b$ from this series as well as their difference spectrum, marking the contribution of the homogeneous freezing mode, are expanded in the top right panel. Spectrum a, which is clearly dominated by the two Christiansen minima of ice, is attributed to a few large ice crystals which nucleated on the Saharan dust particle fraction. Fitting spectrum a in the $1500-800 \mathrm{~cm}^{-1}$ regime yields an ice particle number concentration of $0.6-0.8 \mathrm{~cm}^{-3}$ with $D=8-9 \mu \mathrm{m}$. The exceptionally low percentage of ice-activated dust particles is due to the fact that the most active ice nuclei had been removed by sedimentation in previous activation cycles with the same dust loading, but without sulphuric acid aerosol present. Consequently, the depletion of water vapour by the small number of ice crystals from the first nucleation event was so inefficient that the threshold relative humidity for homogeneous freezing of the $\mathrm{H}_{2} \mathrm{SO}_{4} / \mathrm{H}_{2} \mathrm{O}$ droplets could also be exceeded shortly afterwards, giving rise to the second nucleation event. Beginning with spectrum $b$, there is a rapid increase in the extinction coefficients due to additional ice formation by freezing solution droplets. The difference spectrum $b-a$ clearly bares the signature of smaller, about 3-4 $\mu \mathrm{m}$ sized ice crystals which are formed in the early stage of the homogeneous freezing event. It is obvious from these considerations that spectrum b should be fitted with a bimodal size distribution of ice crystals. But would an unbiased FTIR retrieval, applied to spectrum b, also reveal that the size distribution is bimodal? - The bottom left panel of Fig. 15 shows spectrum $b$ in comparison with the best T-matrix fit results for $\phi=0.7$, employing both a unimodal and a bimodal log-normal size distribution of ice crystals in the retrieval. The bimodal fit closely matches the measured spectrum, and the retrieved size distribution parameters, $N_{1}=6.2 \mathrm{~cm}^{-3}, \sigma_{g, 1}=1.31$, and $D_{1}=3.4 \mu \mathrm{m}$ as well as
$N_{2}=0.58 \mathrm{~cm}^{-3}, \sigma_{g, 2}=1.35$, and $D_{2}=9.8 \mu \mathrm{m}$, nicely reproduce the additional small-particle mode from the homogeneous freezing event. The unimodal fit, which yields a rather broad distribution of smaller ice crystals, $N=13.2 \mathrm{~cm}^{-3}, \sigma_{g}=2.0$, and $D=2.1 \mu \mathrm{m}$, is also acceptable, but slightly poorer than the bimodal one, especially at wavenumbers between 2800 and $2200 \mathrm{~cm}^{-1}$ and in the range $1500-1000 \mathrm{~cm}^{-1}$. Note that spectrum $b$ represents so to speak an ideal scenario to distinguish between uni- and bimodal size distributions in the FTIR retrieval. Two distinct ice crystal modes are known to be present: one mode of larger ice crystals (formed by early deposition nucleation on the mineral dust particles) and a second mode of still small, freshly nucleated ice crystals from the homogeneous freezing of the sulphuric acid droplets. At the early stage of the homogeneous freezing event, both ice crystal modes are clearly separated in size and hold approximately the same ice water content, thus are contributing with equal weight to the overall infrared extinction spectrum. But still, the quality of the bimodal fit is only slightly better than the unimodal fit, indicating that it is extremely difficult to deduce the modality of the size distribution from the FTIR retrieval. This is further evidenced by considering the fit results for spectrum c. When leaving behind the early stage of the homogeneous freezing event (spectrum b), the additional homogeneous ice nucleation mode successively becomes the dominant part of the overall ice cloud. For spectrum c, the ice water content of the homogeneously nucleated ice crystals is already substantially larger than that of the heterogeneously nucleated ice particles, making it even more difficult to clearly identify two modes in the FTIR retrieval. Here, the quality of the unimodal fit $\left(N=20.7 \mathrm{~cm}^{-3}, \sigma_{g}=1.8\right.$, and $\left.D=2.5 \mu \mathrm{m}\right)$ and of the bimodal fit $\left(N_{1}=9.5 \mathrm{~cm}^{-3}, \sigma_{g, 1}=1.26\right.$, and $D_{1}=4.0 \mu \mathrm{m}$ as well as $N_{2}=0.54 \mathrm{~cm}^{-3}, \sigma_{g, 2}=1.23$, and $D_{2}=10.2 \mu \mathrm{m}$ ) is comparable, rendering it entirely impossible to distinguish between uni- and bimodal size distributions and to retrieve the correct number concentration of ice crystals.

\section{Summary and outlook}

The AIDA aerosol and cloud chamber is well suited to quantify the ice nucleation potential of relevant atmospheric aerosol particles over a broad temperature range. We have shown that important properties of the nucleated ice clouds can be readily manipulated by varying the experimental conditions. For instance, the gas temperature, the cooling rate, the number concentration, the type, and the external mixing state of the seed aerosol can be varied independently to control the number concentration, modal composition, and total particle volume of the developing ice cloud. From the large set of AIDA expansion experiments, we have presented selected case studies to assess the retrieval ambiguities when analysing broadband infrared extinction spectra $\left(6000-800 \mathrm{~cm}^{-1}\right)$ of ice crystal clouds in terms of number 
concentration and size distribution of the ice particles. The FTIR retrieval results were compared to independent reference data which had been measured with different optical particle counters (OPCs). Our results may be regarded as a guideline for other laboratory studies of ice nucleation, which frequently have to rely on FTIR extinction spectroscopy without independent information on the accuracy of the retrieval results (e.g. Hung and Martin, 2002).

As a principal conclusion, we want to emphasise that the basic input parameters for the retrieval, i.e., (i) the choice of the model to account for the asphericity of the ice crystals, and (ii) any prior assumption about the modality of the unknown number size distribution of the ice crystals, have to be carefully reconsidered for each single retrieval problem. Erroneous assumptions for both of these two input parameters may cause strong distortions of the actual ice particle number size distribution. For ice crystals with median sizes smaller than about $6 \mu \mathrm{m}$, good agreement between the ice particle number concentrations measured with the OPCs and those deduced from the FTIR spectra was observed when modelling the ice crystals as pure or shape-averaged cylindrical particles of moderate aspect ratios in the range $\phi=0.5-3.0$. However, no information on the actual ice particle shape can be retrieved, (i) because the match between measured and calculated infrared extinction spectra in the $4500-800 \mathrm{~cm}^{-1}$ regime was equally good for all considered particle shapes, and (ii) because the spread of the FTIR fit results in terms of ice crystal number concentrations was always comparable to the uncertainty range of the OPC measurements. For ice crystals with median sizes larger than $6 \mu \mathrm{m}$, however, the application of compact ice crystal shapes in the retrieval (like $\phi=1)$ often gave rise to an overestimation of the actual ice particle number concentration by factors of two or more, far outside the uncertainty range of the OPCs. In most expansion experiments, the diversity of particle habits contributing to the ice clouds gave rise to a near constant optical depth in the non-absorbing wavenumber range above $3600 \mathrm{~cm}^{-1}$, even though the ice particle number size distributions most presumably feature a rather narrow mode width in our experiments. For compact shapes, still exhibiting a pronounced interference pattern in the size-sensitive range of the extinction efficiencies at non-absorbing wavenumbers, such spectral signature can only be reproduced by severely distorting the true number size distribution of the ice particles. As a possible solution to this problem, either cylindrical shapes with aspect ratios significantly different from unity or a suitable shape distribution of pure cylindrical shapes should be used in the retrieval. Alternatively, the wavenumber range of the fit could be restricted to ranges where the dependence of the extinction cross sections on particle shape is less pronounced, as for example in the $1500-800 \mathrm{~cm}^{-1}$ regime. But then, we have also presented a scenario where an exceptionally uniform ice cloud of particles with compact shape parameters was formed, in which case FTIR extinction spectroscopy has proven to be a superior tool for a precise de- termination of the number concentration, size, and shape of the ice crystals by applying the fit to the complete accessible wavenumber range from $6000-800 \mathrm{~cm}^{-1}$. Finally, we have illustrated that it can be extremely difficult to retrieve multimodal number size distributions of ice crystals from infrared spectra. We found that the spectral fit of an observed spectrum, based on a unimodal size distribution, can often be significantly improved by allowing bi- or multimodal size distributions. However, this is more likely a consequence of increasing the degrees of freedom in the retrieval, rather than true evidence that the size distribution is indeed multimodal. One should always be aware of the possibility that the mismatch of the unimodal fit is due to the limited quality of the adopted optical constants and/or the shape representation of the ice crystals, rather than indicating that the size distribution of the ice crystals is bi- or multimodal.

Given that the determination of nucleation rates and ice activation spectra is a main objective of the AIDA expansion experiments, our work has focussed on the performance of the FTIR retrieval for deriving accurate number size distributions of ice crystals. In spite of the frequently encountered severe size/shape ambiguities in these number size distribution retrievals, we want to emphasise that the simultaneously retrieved ice water content of the clouds shows much less variation. In our case studies, the different size/shape representations of the ice crystals usually gave rise to a less than $10 \%$ difference in the retrieved ice water content.

For our present study, we have concentrated on AIDA expansion runs which yielded ice clouds with median crystal sizes smaller than about $15 \mu \mathrm{m}$. This was characteristic for most of the pure ice cloud experiments at $T<237 \mathrm{~K}$ which have been performed so far. More recent experiments (as well as additional chamber studies considered for the future) have been especially designed to generate ice crystals with median sizes of at least $30-40 \mu \mathrm{m}$, i.e., whose shapes can be easily resolved with the CPI cloud particle imager. In addition, a new optical device has been constructed in our own laboratory to measure the scattering phase functions of ice crystals which are simultaneously imaged by a CCD camera with microscope optics. We will perform a detailed instrument inter-comparison with regard to measurements of the size, shape, and number concentration of the ice crystals as well as the ice water content of the generated ice clouds. Benefiting from this work, we will also be able to test the performance of the FTIR retrieval procedure for such larger sized ice crystals, where, in contrast to this study, independent information on the predominant particle shape would be available from the particle imagers.

Acknowledgements. The authors are grateful to all members of the AIDA staff for their continuous support during the numerous successful measurement campaigns on ice nucleation which have been conducted at the chamber facility in the years 2000-2006. We thank R. Cotton and P. Field for the analysis of the SID measurements. The work has been funded by the Helmholtz-Gemeinschaft deutscher Forschungszentren as part of the program "Atmosphere 
and Climate". Part of this work was also funded within the EU Integrated Project "SCOUT-O3".

Edited by: T. Koop

\section{References}

Archuleta, C. M., DeMott, P. J., and Kreidenweis, S. M.: Ice nucleation by surrogates for atmospheric mineral dust and mineral dust/sulfate particles at cirrus temperatures, Atmos. Chem. Phys., 5, 2617-2634, 2005, http://www.atmos-chem-phys.net/5/2617/2005/.

Arnott, W. P., Dong, Y. Y., and Hallett, J., Extinction Efficiency in the Infrared $(2-18 \mu \mathrm{m})$ of Laboratory Ice Clouds - Observations of Scattering Minima in the Christiansen Bands of Ice, Appl. Opt., 34, 541-551, 1995.

Arnott, W. P., Liu, Y., Schmitt, C., and Hallett, J.: The Unreasonable Effectiveness of Mimicking Measured Infrared Extinction by Hexagonal Ice Crystals With Mie Ice Spheres, in: OSA symposium on Optical Remote Sensing of the Atmosphere, pp. 216218, OSA Technical Digest Series (Optical Society of America, Washington D.C.), vol. 5, 1997a.

Arnott, W. P., Schmitt, C., Liu, Y. G., and Hallett, J.: Droplet size spectra and water-vapor concentration of laboratory water clouds: Inversion of Fourier transform infrared (500$5000 \mathrm{~cm}^{-1}$ ) optical-depth measurement, Appl. Opt., 36, 52055216, 1997b.

Bailey, M., and Hallett, J., Growth rates and habits of ice crystals between $-20^{\circ}$ and $-70^{\circ} \mathrm{C}$, J. Atmos. Sci., 61, 514-544, 2004.

Baran, A. J.: Simulation of infrared scattering from ice aggregates by use of a size-shape distribution of circular ice cylinders, Appl. Opt., 42, 2811-2818, 2003.

Baran, A. J.: On the scattering and absorption properties of cirrus cloud, J. Quant. Spectrosc. Radiat. Transfer, 89, 17-36, 2004.

Baran, A. J.: The dependence of cirrus infrared radiative properties on ice crystal geometry and shape of the size-distribution function, Q. J. R. Meteorol. Soc., 131, 1129-1142, 2005.

Benz, S., Megahed, K., Möhler, O., Saathoff, H., Wagner, R., and Schurath, U.: T-dependent rate measurements of homogeneous ice nucleation in cloud droplets using a large atmospheric simulation chamber, J. Photochem. Photobiol. A, 176, 208-217, 2005.

Bohren, C. F. and Huffman, D. R.: Absorption and Scattering of Light by Small Particles, John Wiley \& Sons, Inc., New York, 1983.

Bohren, C. F. and Koh, G.: Forward-scattering corrected extinction by nonspherical particles, Appl. Opt., 24, 1023-1029, 1985.

Clapp, M. L., Miller, R. E., and Worsnop, D. R.: FrequencyDependent Optical Constants of Water Ice Obtained Directly from Aerosol Extinction Spectra, J. Phys. Chem., 99, 63176326, 1995.

Deepak, A. and Box, M. A.: Forwardscattering corrections for optical extinction measurements in aerosol media. 1: Monodispersions, Appl. Opt., 17, 2900-2908, 1978.

Echle, G., von Clarmann, T., and Oelhaf, H.: Optical and microphysical parameters of the Mt. Pinatubo aerosol as determined from MIPAS-B mid-IR limb emission spectra, J. Geophys. Res., 103, 19 193-19211, 1998.

Eremenko, M. N., Zasetsky, A. Y., Boone, C. D., and Sloan, J. J.: Properties of high-altitude tropical cirrus clouds determined from ACE FTS observations, Geophys. Res. Lett., 32, L15S07, doi:10.1029/2005GL022428, 2005.

Field, P. R., Möhler, O., Connolly, P., Krämer, M., Cotton, R., Heymsfield, A. J., and Schnaiter, M.: Some ice nucleation characteristics of Asian and Saharan desert dust, Atmos. Chem. Phys., 6, 2991-3006, 2006,

http://www.atmos-chem-phys.net/6/2991/2006/.

Haag, W., Kärcher, B., Schaefers, S., Stetzer, O., Möhler, O., Schurath, U., Krämer, M., and Schiller, C.: Numerical simulations of homogeneous freezing processes in the aerosol chamber AIDA, Atmos. Chem. Phys., 3, 195-210, 2003, http://www.atmos-chem-phys.net/3/195/2003/.

Hill, S. C., Hill, A. C., and Barber, P. W.: Light scattering by size/shape distributions of soil particles and spheroids, Appl. Opt., 23, 1025-1031, 1984.

Hung, H. M. and Martin, S. T.: Infrared spectroscopic evidence for the ice formation mechanisms active in aerosol flow tubes, Appl. Spectrosc., 56, 1067-1081, 2002.

Kahn, B. H., Eldering, A., Clough, S. A., Fetzer, E. J., Fishbein, E., Gunson, M. R., Lee, S. Y., Lester, P. F., and Realmuto, V. J.: Near micron-sized cirrus cloud particles in high-resolution infrared spectra: An orographic case study, Geophys. Res. Lett., 30(8), 1441, doi:10.1029/2003GL016909, 2003.

Kahn, B. H., Eldering, A., Ghil, M., Bordoni, S., and Clough, S. A.: Sensitivity analysis of cirrus cloud properties from highresolution infrared spectra. Part I: Methodology and synthetic cirrus, J. Climate, 17, 4856-4870, 2004.

Kokhanovsky, A.: Optical properties of terrestrial clouds, Earth-Sci. Rev., 64, 189-241, 2004.

Kokhanovsky, A. A.: Microphysical and optical properties of noctilucent clouds, Earth-Sci. Rev., 71, 127-146, 2005.

Lawson, R. P., Baker, B. A., Schmitt, C. G., and Jensen, T. L.: An overview of microphysical properties of Arctic clouds observed in May and July 1998 during FIRE ACE, J. Geophys. Res., 106, 14 989-15 014, 2001.

Lee, Y. K., Yang, P., Mishchenko, M. I., Baum, B. A., Hu, Y. X., Huang, H. L., Wiscombe, W. J., and Baran, A. J.: Use of circular cylinders as surrogates for hexagonal pristine ice crystals in scattering calculations at infrared wavelengths, Appl. Opt., 42, 2653-2664, 2003.

Libbrecht, K. G. and Yu, H., Crystal growth in the presence of surface melting: supersaturation dependence of the growth of columnar ice crystals, J. Cryst. Growth, 222, 822-831, 2001.

Liu, Y. G., Arnott, W. P., and Hallett, J.: Particle size distribution retrieval from multispectral optical depth: Influences of particle nonsphericity and refractive index, J. Geophys. Res., 104, 31 753-31 762, 1999.

Lynch, D. K., Sassen, K., Starr, D. O., and Stephens, G.: Cirrus, Oxford University Press Inc., USA, 2002.

Mishchenko, M. I.: Light-Scattering by Size Shape Distributions of Randomly Oriented Axially-Symmetrical Particles of a Size Comparable to a Wavelength, Appl. Opt., 32, 4652-4666, 1993.

Mishchenko, M. I. and Travis, L. D.: Capabilities and limitations of a current FORTRAN implementation of the T-matrix method for randomly oriented, rotationally symmetric scatterers, J. Quant. Spectrosc. Radiat. Transfer, 60, 309-324, 1998.

Möhler, O., Büttner, S., Linke, C., Schnaiter, M., Saathoff, H., Stetzer, O., Wagner, R., Krämer, M., Mangold, A., Ebert, V., and Schurath, U.: Effect of Sulphuric Acid Coating on Hetero- 
geneous Ice Nucleation by Soot Aerosol Particles, J. Geophys. Res., 110, D11210, doi:10.1029/2004JD005169, 2005.

Möhler, O., Field, P. R., Connolly, P., Benz, S., Saathoff, H., Schnaiter, M., Wagner, R., Cotton, R., Krämer, M., Mangold, A., and Heymsfield, A. J.: Efficiency of the deposition mode ice nucleation on mineral dust particles, Atmos. Chem. Phys., 6, 3007-3021, 2006, http://www.atmos-chem-phys.net/6/3007/2006/.

Möhler, O., Stetzer, O., Schaefers, S., Linke, C., Schnaiter, M., Tiede, R., Saathoff, H., Krämer, M., Mangold, A., Budz, P., Zink, P., Schreiner, J., Mauersberger, K., Haag, W., Kärcher, B., and Schurath, U.: Experimental investigation of homogeneous freezing of sulphuric acid particles in the aerosol chamber AIDA, Atmos. Chem. Phys., 3, 211-223, 2003, http://www.atmos-chem-phys.net/3/211/2003/.

Moosmüller, H. and Arnott, W. P.: Angular truncation errors in integrating nephelometry, Rev. Sci. Instrum., 74, 3492-3501, 2003.

Press, W. H., Teukolsky, S. A., Vetterling, W. T., and Flannery, B. P.: Numerical Recipes in C: The Art of Scientific Computing, Cambridge University Press, Cambridge, 1992.

Proctor, T. D. and Harris, G. W.: The Turbidity of Suspensions of Irregular Quartz Particles, Aerosol Sci., 5, 81-90, 1974.

Rädel, G., Stubenrauch, C. J., Holz, R., and Mitchell, D. L.: Retrieval of effective ice crystal size in the infrared: Sensitivity study and global measurements from TIROS-N Operational Vertical Sounder, J. Geophys. Res., 108(D9), 4281, doi:10.1029/2002JD002801, 2003.

Rajaram, B., Glandorf, D. L., Curtis, D. B., Tolbert, M. A., Toon, O. B., and Ockman, N.: Temperature-dependent optical constants of water ice in the near infrared: new results and critical review of the available measurements, Appl. Opt., 40, 4449-4462, 2001.

Schmitt, C. G. and Arnott, W. P.: Infrared emission (500$2000 \mathrm{~cm}^{-1}$ ) of laboratory ice clouds, J. Quant. Spectrosc. Radiat. Transfer, 63, 701-725, 1999.

Wagner, R., Benz, S., Möhler, O., Saathoff, H., Schnaiter, M., and Schurath, U.: Mid-infrared extinction spectra and optical constants of supercooled water droplets, J. Phys. Chem. A, 109, 7099-7112, 2005a.

Wagner, R., Bunz, H., Linke, C., Möhler, O., Naumann, K. H., Saathoff, H., Schnaiter, M., and Schurath, U.: Chamber Simulations of Cloud Chemistry: The AIDA Chamber, in: Proceedings of the NATO Advances Research Workshop on Environmental Simulation Chambers: Application to Atmospheric Chemical Processes, held in Zakopane, Poland, from 1 to 4 October 2004, edited by: Barnes, I. and Rudzinski, K. J., Springer, 2006.
Wagner, R., Möhler, O., Saathoff, H., Stetzer, O., and Schurath, U.: Infrared spectrum of nitric acid dihydrate - influence of particle shape, J. Phys. Chem. A, 109, 2572-2581, 2005b.

Walters, P. T.: Pratical applications of inverting spectral turbidity data to provide aerosol size distributions, Appl. Opt., 19, 23532365, 1980.

Warren, S. G.: Optical constants of ice from the ultraviolet to the microwave, Appl. Opt., 23, 1206-1225, 1984.

White, J. U.: Very long optical paths in air, J. Opt. Soc. Am., 66, 411-416, 1976.

Wind, L. and Szymanski, W. W.: Quantification of scattering corrections to the Beer-Lambert law for transmittance measurements in turbid media, Meas. Sci. Technol., 13, 270-275, 2002.

Yang, P., Gao, B. C., Baum, B. A., Hu, Y. X., Wiscombe, W. J., Tsay, S. C., Winker, D. M., and Nasiri, S. L.: Radiative properties of cirrus clouds in the infrared $(8-13 \mu \mathrm{m})$ spectral region, J. Quant. Spectrosc. Radiat. Transfer, 70, 473-504, 2001.

Yang, P., Liou, K. N., and Arnott, W. P.: Extinction efficiency and single-scattering albedo for laboratory and natural cirrus clouds, J. Geophys. Res., 102, 21 825-21 835, 1997.

Yang, P., Wei, H. L., Huang, H. L., Baum, B. A., Hu, Y. X., Kattawar, G. W., Mishchenko, M. I., and Fu, Q.: Scattering and absorption property database for nonspherical ice particles in the near- through far-infrared spectral region, Appl. Opt., 44, 5512 5523, 2005.

Zakharova, N. T. and Mishchenko, M. I.: Scattering properties of needlelike and platelike ice spheroids with moderate size parameters, Appl. Opt., 39, 5052-5057, 2000.

Zasetsky, A. Y., Khalizov, A. F., Earle, M. E., and Sloan, J. J.: Frequency Dependent Complex Refractive Indices of Supercooled Liquid Water and Ice Determined from Aerosol Extinction Spectra, J. Phys. Chem. A, 109, 2760-2764, 2005.

Zasetsky, A. Y., Khalizov, A. F., and Sloan, J. J.: Characterization of atmospheric aerosols from infrared measurements: simulations, testing, and applications, Appl. Opt., 43, 5503-5511, 2004. 